\title{
Monitoring Risk: Tick and Borrelia burgdorferi Public Participatory Surveillance in the Canadian Maritimes, 2012-2020
}

\author{
Julie Lewis ${ }^{1,2, \dagger}$, Andrea M. Kirby ${ }^{1, \dagger}$, Kami Dawn Harris ${ }^{1,3}$, Cory L. Filiaggi ${ }^{1,4}$, Alexandra Foley-Eby ${ }^{1,5}$, \\ Malcolm Mann ${ }^{1,6}$, David Lieske ${ }^{6}$ and Vett K. Lloyd ${ }^{1, *}$
}

1 Department of Biology, Mount Allison University, Sackville, NB E4L 1G7, Canada; jlewis@mta.ca (J.L.); amkirby@mta.ca (A.M.K.); sgreenwood@upei.ca (K.D.H.); corey.filiaggi@iwk.nshealth.ca (C.L.F.); fxu874@usask.ca (A.F.-E.); mgmann@mta.ca (M.M.)

2 Département de Chimie et Biochimie, Université de Moncton, Moncton, NB E1A 3E9, Canada

3 Department of Pathology and Microbiology, Atlantic Veterinary College, University of Prince Edward Island, Charlottetown, PE C1A 4P3, Canada

4 IWK Health Centre, Halifax, NS B3K 6R8, Canada

5 Department of Veterinary Microbiology, University of Saskatchewan, Saskatoon, SK S7N 5B4, Canada

6 Department of Geography and Environment, Mount Allison University, Sackville, NB E4L 1G7, Canada; dlieske@mta.ca

* Correspondence: vlloyd@mta.ca; Tel.: +1-506-364-2509

+ Contributed equally to this work.

\section{check for} updates

Citation: Lewis, J.; Kirby, A.M.; Harris, K.D.; Filiaggi, C.L.; Foley-Eby, A.; Mann, M.; Lieske, D.; Lloyd, V.K. Monitoring Risk: Tick and Borrelia burgdorferi Public Participatory Surveillance in the Canadian Maritimes, 2012-2020. Pathogens 2021, 10, 1284. https: / / doi.org/10.3390/ pathogens10101284

Academic Editor: Alejandro Cabezas-Cruz

Received: 30 August 2021 Accepted: 30 September 2021 Published: 6 October 2021

Publisher's Note: MDPI stays neutral with regard to jurisdictional claims in published maps and institutional affiliations.

Copyright: (c) 2021 by the authors. Licensee MDPI, Basel, Switzerland. This article is an open access article distributed under the terms and conditions of the Creative Commons Attribution (CC BY) license (https:// creativecommons.org/licenses/by/ $4.0 /)$.
Abstract: Ticks are vectors of many diseases, including Lyme disease (Ld). Lyme disease is an emerging disease in Canada caused by infection with the Lyme borreliosis $(\mathrm{Lb})$ members of the Borrelia genus of spirochaete bacteria, of which Borrelia burgdorferi is regionally the most prevalent. The primary tick vector in central and eastern Canada, Ixodes scapularis, is increasing in numbers and in the geographical extent of established populations. This study documents the distribution of ticks recovered by passive surveillance, and their B. burgdorferi infection prevalence, in three Canadian Maritime provinces from 2012-2020. These regions represent areas in which tick populations are widely established, establishing, and considered non-established. Using a community science approach by partnering with veterinarians and members of the public, we collected over 7000 ticks from the 3 provinces. The three species found most often on companion animals and humans were I. scapularis $(76.9 \%)$, Ixodes cookei $(10.4 \%)$ and Dermacentor variabilis $(8.9 \%)$. The most common hosts were dogs $(60.5 \%)$, cats $(16.8 \%)$ and humans $(17.6 \%)$. As is typical of passive surveillance tick collections, the majority of ticks recovered were adult females; for I. scapularis $90.2 \%, 5.3 \%, 3.9 \%$ and $0.6 \%$ of the total of 5630 ticks recovered for this species were adult females, adult males, nymphs and larvae, respectively. The majority of B. burgdorferi-infected ticks were I. scapularis, as expected. Borrelia infection prevalence in I scapularis was higher in Nova Scotia $(20.9 \%)$, the province with the most endemic regions, than New Brunswick (14.1\%) and Prince Edward Island (9.1\%), provinces thought to have established and non-established tick populations, respectively. The province-wide Borrelia infection prevalence generally increased in these latter tow provinces over the course of the study. The host did not have a significant effect on B. burgdorferi infection prevalence; I. scapularis ticks from dogs, cats, humans was, $13.3 \%(n=3622), 15.6 \%(n=817), 17.9 \%(n=730)$, respectively. No I. scapularis larvae were found infected $(\mathrm{n}=33)$ but B. burgdorferi was detected in $14.8 \%$ of both adults $(n=5140)$ and nymphs $(n=215)$. The incidence of $B$. burgdorferi infection also did not differ by engorgement status $15.0 \%(n=367), 15.1 \%(n=3101)$ and $14.4 \%(n=1958)$ of non-engorged, engorged and highly engorged ticks, respectively, were infected. In New Brunswick, at the advancing front of tick population establishment, the province-wide infection percentages generally increased over the nine-year study period and all health district regions showed increased tick recoveries and a trend of increased percentages of Borrelia-infected ticks over the course of the study. Within New Brunswick, tick recoveries but not Borrelia infection prevalence were significantly different from endemic and non-endemic regions, suggesting cryptic endemic regions existed prior to their designation as a risk area. Over the 9 years of the study, tick recoveries increased in New Brunswick, the primary study 
region, and I. scapularis recoveries spread northwards and along the coast, most but not all new sites of recoveries were predicted by climate-based models, indicating that ongoing tick surveillance is necessary to accurately detect all areas of risk. Comparison of tick recoveries and public health risk areas indicates a lag in identification of risk areas. Accurate and timely information on tick distribution and the incidence of Borrelia and other infections are essential for keeping the public informed of risk and to support disease prevention behaviors.

Keywords: ticks; surveillance; Ixodes scapularis; Ixodes cookei; Dermacentor variabilis; Canada; Borrelia

\section{Introduction}

Ticks are obligate blood-feeding ectoparasites that are able to transmit a greater array of pathogens than any other arthropod [1]. As ticks feed, nutrients from the host's blood are absorbed, excess water is removed by the tick salivary glands and secreted back into the host [2]. During this process, a variety of microorganisms including bacteria, viruses, protozoa, fungi, and nematodes, some of which are pathogenic, can be transferred to the host $[3,4]$. In this way, ticks can vector many disparate pathogens, producing a variety of diseases. In temperate regions of the world, the most common of these is $\mathrm{Ld}$, or $\mathrm{Lb}$. Ld is caused by some species of the Lb group, or Borrelia burgdorferi sensu lato complex [5], although only Borrelia burgdorferi sensu stricto is routinely monitored in North America [6,7]. Ld is marked by a panoply of multisystemic symptoms that affect the musculoskeletal, respiratory, circulatory, nervous, and other systems, which can have debilitating or in some cases fatal, health consequences [7-11].

In eastern Canada, the tick species that most often parasitize humans and companion animals include Ixodes scapularis (Say, 1821) also known as the blacklegged or deer tick, Ixodes cookei (Packard, 1869) or the groundhog/woodchuck tick, and Dermacentor variabilis (Say, 1821) or the American dog tick [12]. All of these ticks can vector a variety of pathogens including Borrelia spirocheates [12], which are efficiently vectored by I. scapularis [13] and, possibly less efficiently, by I. cookei $[14,15]$. Both species also transmit other pathogens including the Powassan virus/Deer Tick Virus, which causes an infection of the central nervous system resulting in encephalitis and meningitis [16]. D. variabilis, the American dog tick or wood tick, is not thought to be a competent vector of $B$. burgdorferi but can transmit Francisella tularensis, the bacteria responsible for tularemia, Rickettsia rickettsii, the bacteria responsible for Rocky Mountain spotted fever, Ehrlichia chaffeensis, the bacteria responsible for Ehrlichiosis, Anaplasma marginale, the bacteria responsible for Anaplasmosis or tick-born fever in a variety of mammals, and likely related pathogens [17-20].

The tick species that parasitize humans and companion animals do so because they are generalist feeders, so are not overly meticulous in choosing their hosts [12]. As a consequence, many mammalian host species are available to support the expanding populations of I. scapularis, D. variabilis and other introduced tick species [21]. Further, many of these species are effective reservoir hosts for B. burgdorferi, thus perpetuating the infection cycle. Reservoir species in the Canadian Maritimes include a wide variety of rodents such as deer mice and white-footed mice, shrews, squirrels, chipmunks, and wood rats [6,22]. Some bird species are also effective reservoirs [23], in addition to their important role in tick dispersal. Each spring, an estimated 50 to 175 million I. scapularis are dispersed into Canada by migratory birds [24], with some species carrying I. scapularis long distances northwards [25-27]. Once introduced, both biotic and abiotic factors influence the proportion of ticks able to feed and survive long enough to reproduce and establish new populations [28]. Surveillance efforts have revealed a northward expansion of established I. scapularis and $D$. variabilis populations in response to complex and inter-related changes in climate, mammal and bird migrations, rodent populations, landscape fragmentation and the resultant effect on biodiversity, the nature of vegetation and forests and foliage 
type, wildlife management practices, human demographics, and other anthropomorphic factors $[21,29,30]$.

Humans and companion animals have lifestyles that make the repeated contact with ticks, needed to maintain tick or Borrelia populations, unlikely, and are, therefore, considered accidental hosts rather than reservoirs. Thus, barring congenital transmission of Borrelia [31], humans are not ecologically relevant in perpetuating Borrelia in the environment. Nevertheless, Borrelia infections can result in Ld and are of considerable importance in both human and veterinary medicine. Tick surveillance plays an important role in disease diagnosis and subsequent treatment; clinical and serological evidence is considered in light of a history of exposure to known tick endemic areas [32]. Without known exposure, positive serology results risk being discounted by invocation of pre-test probability considerations [33].

Tick surveillance allows monitoring of I. scapularis distribution and B. burgdorferi infection prevalence. How the surveillance is performed affects its sensitivity. Active surveillance involves capturing ticks from the environment, whereas passive surveillance involves collecting ticks from humans and companion, agricultural, or wild animals [34]. Both collection methods have strengths and weaknesses [34]. Active surveillance suffers from low sensitivity. It is less efficient for a single team to find ticks in the field during a short site visit than having veterinarians and members of the public involved in the collection process over a period of years. Additionally, ticks are only captured from the environment when actively questing for a blood meal and appropriate conditions for this activity may not occur at the time of site visit. Finally, field collection of ticks relies on ticks being captured by an inert "tick drag" that does not mimic a host in a biologically meaningful manner, although adding a source of $\mathrm{CO}_{2}$ or host body odor to the sheet has been shown to improve tick collection for a European tick species [35]. All these factors contribute to reduced sensitivity of this surveillance method. This can lead to an underestimation of tick populations, particularly in areas with low tick density as occurs in areas with emerging tick populations, making this an unsuitable surveillance method in such situations. However, active surveillance does permit precise geographical location of recovered ticks, recording of ecosystem variables and has the advantage of allowing direct comparison of tick density between populations as search effort is not influenced by proximity to human communities so it is valuable for ecological studies in endemic areas [36]. Passive collection, often involving a community science approach, is able to collect more ticks over a larger geographic area with fewer logistical challenges than active surveillance [37]. Passive surveillance has been criticized for being too sensitive as it can recover both endemic ticks from local populations and adventitious ticks dropped outside of endemic areas by migratory animals [34]. Nonetheless, as the health risk from ticks stems from both home-grown ticks in endemic locations and introduced adventitious ticks [36], this is, in fact, a strength of passive surveillance in the context of risk assessment. Passive surveillance also provides the capacity for sensitive monitoring of the continually evolving endemic areas. Ultimately, the impact of these relative advantages and disadvantages depends on the research question being addressed, however in a public health setting, the lower cost and increased sensitivity associated with passive surveillance are considerable advantages.

Comprehensive surveillance in areas outside of known endemic regions is critical for monitoring the continually evolving locations and size of tick populations [38]. The three Canadian Maritime provinces of Nova Scotia (NS), New Brunswick (NB) and Prince Edward Island (PEI), while geographically connected, differ in respect to the establishment of populations of the introduced I. scapularis, I. cookei and D. variabilis tick species. Nova Scotia is the southernmost province and, during the study period of 2012-2020, known endemic and Ld risk areas expanded from the southwest of the province across the entire province [39]. During the same time period, the known endemic and risk areas in New Brunswick expanded from two localized populations in the southwest to the southern third of the province $[40,41]$. Prince Edward Island has been considered to have no endemic tick 
populations, although ticks that are presumably adventitious have been reported on the island since 1989 [42]. In this study, we report passive surveillance tick recoveries and $B$. burgdorferi infection prevalence from these three neighboring provinces during a 9-year period from 2012-2020. Our results show an expanding risk for Ld outside of known and suspected endemic sites, particularly in riparian and coastal regions.

\section{Results}

\subsection{Tick Submissions}

The number of ticks submitted over the course of the study increased over time; 365 , $588,774,792,1026,1350,814,1181$, and 433 were submitted from 2012-2020, respectively, for a total of 7323 (Table 1). The reduced number of submissions in 2020 corresponds with transition to cost-recovery versus free tick testing. The most commonly submitted tick species, all years combined, were I. scapularis $(76.9 \% ; 5630 / 7323)$, I. cookei $(10.4 \%$; $761 / 7323)$ and D. variabilis $(8.9 \% ; 655 / 7323)$ (Table 1$)$. Other less frequently recovered species included Haemaphysalis leporispalustris (Packard, 1869) (rabbit tick), Ixodes muris (Bishopp and Smith, 1937) (mouse tick), Dermacentor albipictus (Packard, 1869) (moose tick), Ixodes marxi (Banks, 1908) (squirrel tick), Ixodes uriae (White, 1852) (seabird tick), Rhipicephalus sanguineus (Latreille, 1806) (brown dog tick) which collectively comprised 0.9\% of recoveries $(66 / 7323)$. As expected for passive surveillance, and consistent with similar studies [43-46]; most submissions were of adult females, for all tick species (Table 1). For $I$. scapularis $90.2 \%, 5.3 \%, 3.9 \%$ and $0.6 \%$ of the total of 5630 ticks recovered for this species were adult females, adult males, nymphs and larvae, respectively. For I. cookei, 54.4\%, 1.3\%, 35\% and $9.3 \%$ of the 761 recovered ticks of this species were adult females, adult males, nymphs and larvae, respectively. For D. variabilis, 62.3\%, 33.6\%, 2.6\% and 1.5\% of the 655 recovered ticks of this species were adult females, adult males, nymphs and larvae, respectively. The most common hosts contributing ticks to this study were dogs $(60.5 \%)$, cats $(16.8 \%)$ and humans (17.6\%) (Table 1). Additionally, ticks were also recovered unfed from the field, after detaching from an unknown host when fully engorged, from unspecified hosts and also from wildlife and agricultural hosts (cows, pigs, horses, groundhogs, coyotes, foxes, moose, bear, deer, rabbits, hares, seabirds, songbirds, mice, voles, skunks, squirrels, raccoons and shrews) (Table 1 ). This study was promoted exclusively in New Brunswick and $78.9 \%$ of ticks came from the province. However, ticks were also received from the other Canadian provinces including Nova Scotia (17.2\%), Ontario (1.1\%), Prince Edward Island (1.0\%), Quebec (0.2\%), Alberta (0.2\%), British Columbia $(<0.1 \%)$, Manitoba $(<0.1 \%)$, Saskatchewan $(<0.1 \%)$ (Table 2$)$. Additionally, ticks were received from other countries in North America, South America, Europe, Africa, Asia and Australia (0.9\%) and from unspecified locations $(0.7 \%)$. Submissions from Prince Edward Island were low in the earlier years of this study, reflecting a local tick surveillance program led by the Island veterinary community [44]. When the program was discontinued, tick submissions to this study increased and a targeted passive surveillance program on Prince Edward Island in 2016 and 2017 retrieved 445 ticks, 97.8\% of which were I. scapularis [47]. This suggests that the low recoveries from Prince Edward Island reflects lack of local targeted surveillance rather than lack of ticks. 
Table 1. Tick donation by species and life stages between 2012 and 2020 .

\begin{tabular}{|c|c|c|c|c|c|c|c|c|c|c|c|c|c|c|c|c|c|c|c|c|c|c|c|c|c|c|c|}
\hline & & \multicolumn{5}{|c|}{ I. scapularis } & \multicolumn{5}{|c|}{ I. cookei } & \multicolumn{4}{|c|}{ D. variabilis } & \multicolumn{6}{|c|}{ Unknown $^{1}$} & \multicolumn{4}{|c|}{ Other Tick Species $^{2}$} & \multirow[b]{2}{*}{ 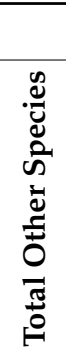 } & \multirow[b]{2}{*}{ 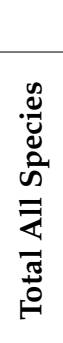 } \\
\hline & & 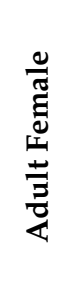 & 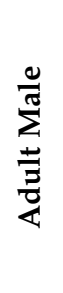 & 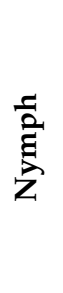 & 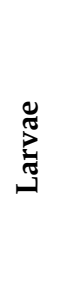 & 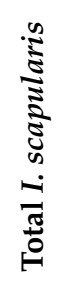 & 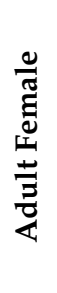 & 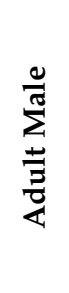 & $\underset{\text { Z }}{\stackrel{\text { Z }}{\mathbf{Z}}}$ & 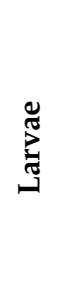 & 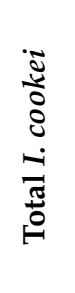 & 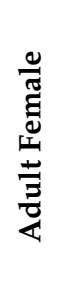 & $\frac{\frac{0}{\pi}}{\sum_{\pi}^{\pi}}$ & 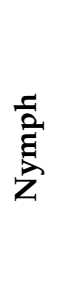 & 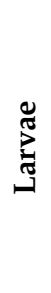 & 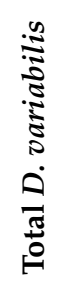 & 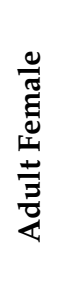 & 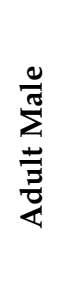 & $\frac{\text { 告 }}{\text { Z }}$ & 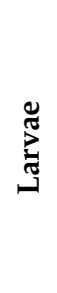 & 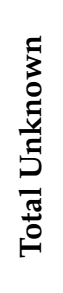 & 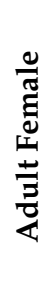 & 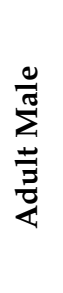 & $\underset{\text { Z }}{\stackrel{a}{a}}$ & 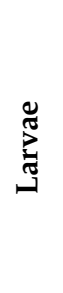 & & \\
\hline \multirow{5}{*}{ 콜 } & Cat & 63 & 4 & 3 & 0 & 70 & 16 & 0 & 0 & 0 & 16 & 0 & 0 & 0 & 0 & 0 & 11 & 2 & 0 & 0 & 13 & 0 & 0 & 0 & 0 & 0 & 99 \\
\hline & Human & 13 & 1 & 0 & 20 & 34 & 3 & 0 & 0 & 0 & 3 & 3 & 1 & 0 & 0 & 4 & 2 & 0 & 0 & 0 & 2 & 0 & 0 & 0 & 0 & 0 & 43 \\
\hline & Other host ${ }^{3}$ & 0 & 0 & 0 & 0 & 0 & 3 & 0 & 2 & 0 & 5 & 0 & 0 & 0 & 0 & 0 & 0 & 0 & 1 & 0 & 1 & 0 & 0 & 0 & 0 & 0 & 6 \\
\hline & Undetermined $^{4}$ & 8 & 2 & 0 & 0 & 10 & 0 & 0 & 0 & 0 & 0 & 0 & 0 & 0 & 0 & 0 & 0 & 0 & 0 & 0 & 0 & 0 & 0 & 0 & 0 & 0 & 10 \\
\hline & Total 2012 & 251 & 15 & 5 & 20 & 291 & 36 & 0 & 2 & 0 & 38 & 6 & 5 & 0 & 0 & 11 & 21 & 3 & 1 & 0 & 25 & 0 & 0 & 0 & 0 & 0 & 365 \\
\hline \multirow{5}{*}{$\stackrel{m}{\stackrel{\infty}{\sim}}$} & Dog & 311 & 15 & 4 & 0 & 330 & 23 & 0 & 4 & 6 & 33 & 15 & 4 & 0 & 0 & 19 & 3 & 1 & 0 & 4 & 8 & 2 & 1 & 0 & 0 & 1 & 391 \\
\hline & Human & 26 & 1 & 3 & 6 & 36 & 6 & 6 & 0 & 0 & 12 & 17 & 2 & 2 & 5 & 26 & 1 & 0 & 0 & 2 & 3 & 1 & 0 & 0 & 0 & 0 & 77 \\
\hline & Other host ${ }^{3}$ & 1 & 0 & 0 & 0 & 1 & 0 & 0 & 0 & 0 & 0 & 0 & 0 & 0 & 0 & 0 & 0 & 0 & 0 & 0 & 0 & 0 & 0 & 0 & 0 & 0 & 1 \\
\hline & Undetermined $^{4}$ & 4 & 0 & 1 & 0 & 5 & 0 & 0 & 0 & 0 & 0 & 13 & 0 & 0 & 0 & 13 & 0 & 0 & 0 & 0 & 0 & 0 & 0 & 0 & 0 & 0 & 18 \\
\hline & Total 2013 & 385 & 19 & 10 & 6 & 420 & 77 & 6 & 5 & 6 & 94 & 46 & 6 & 2 & 5 & 59 & 4 & 1 & 0 & 6 & 11 & 3 & 1 & 0 & 0 & 1 & 585 \\
\hline \multirow{6}{*}{$\underset{\sim}{\vec{\sim}}$} & Dog & 426 & 18 & 5 & 0 & 449 & 59 & 2 & 4 & 14 & 79 & 12 & 2 & 0 & 0 & 14 & 22 & 0 & 0 & 6 & 28 & 0 & 0 & 0 & 0 & 0 & 570 \\
\hline & Cat & 71 & 3 & 2 & 0 & 76 & 23 & 0 & 11 & 5 & 39 & 0 & 0 & 0 & 0 & 0 & 6 & 0 & 0 & 0 & 6 & 0 & 0 & 0 & 0 & 0 & 121 \\
\hline & Human & 33 & 4 & 5 & 0 & 42 & 1 & 0 & 4 & 0 & 5 & 10 & 6 & 0 & 0 & 16 & 1 & 0 & 0 & 0 & 1 & 0 & 0 & 0 & 0 & 0 & 64 \\
\hline & Other host ${ }^{3}$ & 5 & 1 & 0 & 0 & 6 & 0 & 0 & 0 & 0 & 0 & 0 & 1 & 0 & 0 & 1 & 0 & 0 & 0 & 0 & 0 & 0 & 0 & 0 & 0 & 0 & 7 \\
\hline & Undetermined $^{4}$ & 8 & 0 & 1 & 0 & 9 & 0 & 0 & 1 & 0 & 1 & 1 & 0 & 0 & 0 & 1 & 0 & 0 & 1 & 0 & 1 & 0 & 0 & 0 & 0 & 0 & 12 \\
\hline & Total 2014 & 543 & 26 & 13 & 0 & 582 & 83 & 2 & 20 & 19 & 124 & 23 & 9 & 0 & 0 & 32 & 29 & 0 & 1 & 6 & 36 & 0 & 0 & 0 & 0 & 0 & 774 \\
\hline
\end{tabular}


Table 1. Cont.

\begin{tabular}{|c|c|c|c|c|c|c|c|c|c|c|c|c|c|c|c|c|c|c|c|c|c|c|c|c|c|c|c|}
\hline & & \multicolumn{5}{|c|}{ I. scapularis } & \multicolumn{5}{|c|}{ I. cookei } & \multicolumn{4}{|c|}{ D. variabilis } & \multicolumn{6}{|c|}{ Unknown $^{1}$} & \multicolumn{4}{|c|}{ Other Tick Species $^{2}$} & \multirow[b]{2}{*}{ 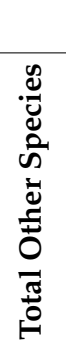 } & \multirow[b]{2}{*}{ 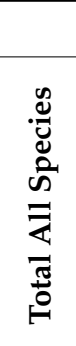 } \\
\hline & & 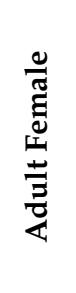 & $\frac{\sum_{\pi}^{\frac{\pi}{\pi}}}{\frac{0}{3}}$ & $\begin{array}{l}\frac{2}{2} \\
\text { Z्र } \\
\text { Z }\end{array}$ & 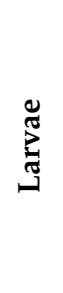 & 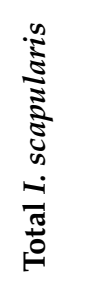 & 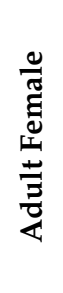 & 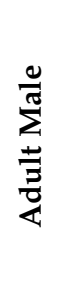 & $\frac{\text { a }}{\mathfrak{z}}$ & 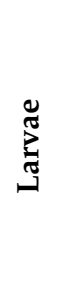 & 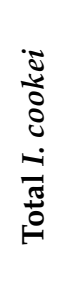 & 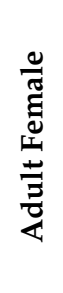 & 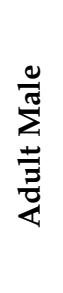 & 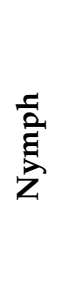 & 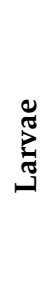 & 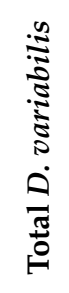 & 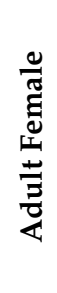 & $\frac{\frac{0}{\pi}}{\frac{\pi}{2}}$ & $\begin{array}{l}\text { 竞 } \\
\text { 乙 }\end{array}$ & 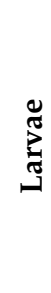 & 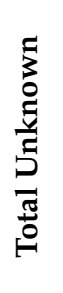 & 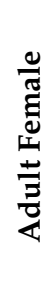 & $\frac{\sum_{\pi}^{\frac{\pi}{\pi}}}{\frac{0}{3}}$ & 总 & 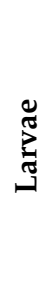 & & \\
\hline \multirow{5}{*}{$\stackrel{\llcorner 0}{\stackrel{\sim}{\sim}}$} & Cat & 76 & 2 & 12 & 0 & 90 & 19 & 0 & 19 & 0 & 38 & 0 & 0 & 0 & 0 & 0 & 1 & 0 & 1 & 0 & 2 & 0 & 3 & 0 & 0 & 3 & 133 \\
\hline & Human & 29 & 3 & 14 & 0 & 46 & 2 & 0 & 4 & 0 & 6 & 16 & 18 & 2 & 0 & 36 & 1 & 1 & 3 & 0 & 5 & 0 & 0 & 0 & 0 & 0 & 93 \\
\hline & Other host ${ }^{3}$ & 1 & 0 & 0 & 0 & 1 & 0 & 0 & 0 & 0 & 0 & 3 & 2 & 0 & 0 & 5 & 1 & 0 & 3 & 0 & 4 & 0 & 0 & 0 & 0 & 0 & 10 \\
\hline & Undetermined $^{4}$ & 11 & 0 & 2 & 0 & 13 & 0 & 0 & 0 & 0 & 0 & 1 & 1 & 0 & 0 & 2 & 1 & 0 & 0 & 0 & 1 & 0 & 0 & 0 & 0 & 0 & 16 \\
\hline & Total 2015 & 517 & 14 & 37 & 4 & 572 & 54 & 0 & 39 & 1 & 94 & 58 & 39 & 2 & 0 & 99 & 11 & 1 & 11 & 0 & 23 & 1 & 3 & 0 & 0 & 3 & 791 \\
\hline \multirow{5}{*}{$\begin{array}{l}\text { o } \\
\stackrel{\sim}{\sim}\end{array}$} & Dog & 520 & 24 & 6 & 0 & 550 & 14 & 0 & 8 & 12 & 34 & 12 & 3 & 1 & 0 & 16 & 14 & 5 & 2 & 0 & 21 & 9 & 1 & 0 & 0 & 1 & 622 \\
\hline & Human & 77 & 6 & 8 & 1 & 92 & 2 & 0 & 2 & 0 & 4 & 24 & 13 & 0 & 0 & 37 & 2 & 0 & 0 & 0 & 2 & 0 & 0 & 0 & 0 & 0 & 135 \\
\hline & Other host ${ }^{3}$ & 7 & 1 & 0 & 0 & 8 & 0 & 0 & 0 & 0 & 0 & 11 & 0 & 0 & 0 & 11 & 1 & 0 & 0 & 0 & 1 & 0 & 0 & 0 & 0 & 0 & 20 \\
\hline & Undetermined ${ }^{4}$ & 18 & 9 & 0 & 0 & 27 & 0 & 0 & 0 & 0 & 0 & 2 & 0 & 0 & 0 & 2 & 0 & 1 & 0 & 0 & 1 & 0 & 0 & 0 & 0 & 0 & 30 \\
\hline & Total 2016 & 754 & 46 & 20 & 2 & 822 & 36 & 0 & 32 & 23 & 91 & 53 & 16 & 1 & 0 & 70 & 21 & 6 & 3 & 3 & 33 & 9 & 1 & 0 & 0 & 1 & 1017 \\
\hline \multirow{6}{*}{ 홍 } & Dog & 607 & 44 & 5 & 1 & 657 & 7 & 0 & 26 & 7 & 40 & 29 & 13 & 0 & 0 & 42 & 18 & 7 & 8 & 0 & 33 & 4 & 3 & 1 & 0 & 4 & 776 \\
\hline & Cat & 140 & 9 & 10 & 0 & 159 & 10 & 0 & 16 & 0 & 26 & 1 & 0 & 0 & 0 & 1 & 10 & 1 & 2 & 0 & 13 & 0 & 0 & 0 & 0 & 0 & 199 \\
\hline & Human & 160 & 7 & 38 & 0 & 205 & 3 & 0 & 6 & 0 & 9 & 27 & 32 & 0 & 1 & 60 & 4 & 2 & 6 & 0 & 12 & 1 & 0 & 1 & 0 & 1 & 287 \\
\hline & Other host ${ }^{3}$ & 8 & 0 & 2 & 0 & 10 & 0 & 0 & 8 & 1 & 9 & 0 & 0 & 1 & 0 & 1 & 1 & 0 & 0 & 0 & 1 & 2 & 0 & 0 & 0 & 0 & 21 \\
\hline & Undetermined $^{4}$ & 37 & 4 & 1 & 0 & 42 & 0 & 0 & 2 & 0 & 2 & 8 & 5 & 0 & 1 & 14 & 2 & 0 & 0 & 0 & 2 & 0 & 0 & 0 & 0 & 0 & 60 \\
\hline & Total 2017 & 952 & 64 & 56 & 1 & 1073 & 20 & 0 & 58 & 8 & 86 & 65 & 50 & 1 & 2 & 118 & 35 & 10 & 16 & 0 & 61 & 7 & 3 & 2 & 0 & 5 & 1343 \\
\hline
\end{tabular}


Table 1. Cont.

\begin{tabular}{|c|c|c|c|c|c|c|c|c|c|c|c|c|c|c|c|c|c|c|c|c|c|c|c|c|c|c|c|}
\hline & & \multicolumn{4}{|c|}{ I. scapularis } & \multicolumn{6}{|c|}{ I. cookei } & \multicolumn{4}{|c|}{ D. variabilis } & \multicolumn{6}{|c|}{ Unknown $^{1}$} & \multicolumn{4}{|c|}{ Other Tick Species ${ }^{2}$} & \multirow[b]{2}{*}{ 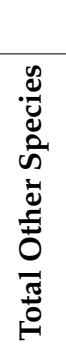 } & \multirow[b]{2}{*}{ 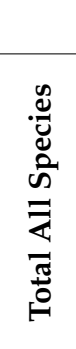 } \\
\hline & & 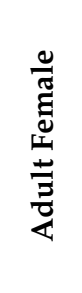 & 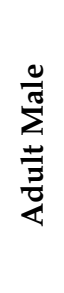 & 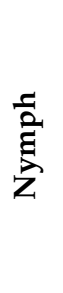 & 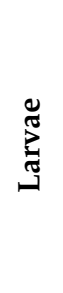 & 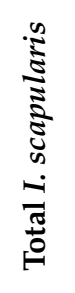 & 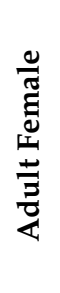 & 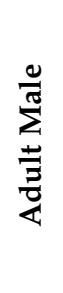 & $\frac{\mathfrak{z}}{\mathfrak{z}}$ & 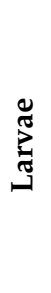 & 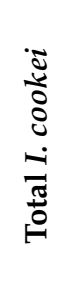 & 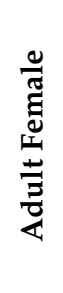 & 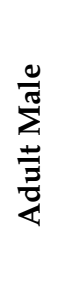 & 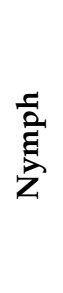 & 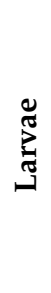 & 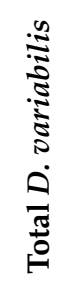 & 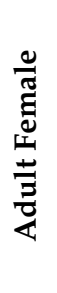 & 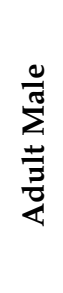 & $\frac{\text { a }}{\text { Z }}$ & 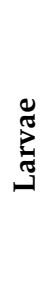 & 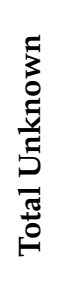 & 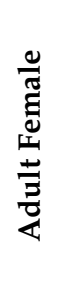 & $\frac{\frac{0}{\pi}}{\sum_{\frac{\pi}{2}}}$ & $\underset{\mathrm{Z}}{\stackrel{a}{2}}$ & 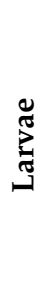 & & \\
\hline \multirow{5}{*}{$\stackrel{\infty}{\stackrel{\infty}{\sim}}$} & Cat & 98 & 2 & 17 & 0 & 117 & 20 & 0 & 26 & 1 & 47 & 3 & 0 & 0 & 0 & 3 & 0 & 0 & 0 & 0 & 0 & 2 & 0 & 0 & 0 & 0 & 167 \\
\hline & Human & 71 & 12 & 18 & 0 & 101 & 5 & 0 & 11 & 0 & 16 & 41 & 22 & 0 & 1 & 64 & 0 & 0 & 0 & 0 & 0 & 15 & 0 & 2 & 0 & 2 & 183 \\
\hline & Other host ${ }^{3}$ & 5 & 0 & 0 & 0 & 5 & 1 & 0 & 0 & 0 & 1 & 0 & 0 & 0 & 0 & 0 & 0 & 0 & 0 & 0 & 0 & 0 & 1 & 0 & 0 & 1 & 7 \\
\hline & Undetermined $^{4}$ & 11 & 0 & 0 & 0 & 11 & 0 & 0 & 0 & 0 & 0 & 3 & 0 & 8 & 0 & 11 & 1 & 0 & 0 & 0 & 1 & 0 & 0 & 0 & 0 & 0 & 23 \\
\hline & Total 2018 & 494 & 41 & 37 & 0 & 572 & 45 & 2 & 53 & 6 & 106 & 67 & 35 & 8 & 1 & 111 & 1 & 1 & 0 & 0 & 2 & 17 & 4 & 2 & 0 & 6 & 797 \\
\hline \multirow{5}{*}{$\stackrel{\curvearrowright}{\vec{\curvearrowright}}$} & Dog & 471 & 29 & 2 & 0 & 502 & 24 & 0 & 9 & 2 & 35 & 28 & 15 & 1 & 0 & 44 & 0 & 1 & 0 & 0 & 1 & 2 & 1 & 1 & 0 & 2 & 584 \\
\hline & Human & 217 & 7 & 31 & 0 & 255 & 4 & 0 & 7 & 0 & 11 & 39 & 32 & 2 & 0 & 73 & 0 & 0 & 1 & 1 & 2 & 2 & 0 & 3 & 0 & 3 & 344 \\
\hline & Other host ${ }^{3}$ & 4 & 0 & 0 & 0 & 4 & 3 & 0 & 0 & 0 & 3 & 1 & 1 & 0 & 0 & 2 & 0 & 0 & 0 & 0 & 0 & 2 & 0 & 0 & 0 & 0 & 9 \\
\hline & Undetermined ${ }^{4}$ & 50 & 7 & 2 & 0 & 59 & 0 & 0 & 2 & 0 & 2 & 8 & 5 & 0 & 1 & 14 & 2 & 0 & 0 & 0 & 2 & 0 & 0 & 0 & 0 & 0 & 77 \\
\hline & Total 2019 & 857 & 45 & 37 & 0 & 939 & 45 & 0 & 39 & 6 & 90 & 76 & 55 & 3 & 1 & 135 & 2 & 2 & 1 & 1 & 6 & 6 & 1 & 4 & 0 & 5 & 1175 \\
\hline \multirow{6}{*}{ ત્ి } & Dog & 243 & 23 & 0 & 0 & 266 & 15 & 0 & 6 & 0 & 21 & 8 & 4 & 0 & 0 & 12 & 2 & 0 & 5 & 0 & 7 & 0 & 1 & 0 & 0 & 1 & 307 \\
\hline & Cat & 23 & 1 & 2 & 0 & 26 & 1 & 0 & 8 & 1 & 10 & 0 & 0 & 0 & 0 & 0 & 2 & 0 & 5 & 0 & 7 & 0 & 0 & 0 & 0 & 0 & 43 \\
\hline & Human & 46 & 6 & 2 & 0 & 54 & 1 & 0 & 3 & 0 & 4 & 6 & 1 & 0 & 1 & 8 & 0 & 0 & 0 & 0 & 0 & 1 & 0 & 0 & 0 & 0 & 66 \\
\hline & Other host ${ }^{3}$ & 4 & 0 & 0 & 0 & 4 & 0 & 0 & 0 & 0 & 0 & 0 & 0 & 0 & 0 & 0 & 0 & 0 & 0 & 0 & 0 & 0 & 0 & 0 & 0 & 0 & 4 \\
\hline & Undetermined 4 & 9 & 0 & 0 & 0 & 9 & 1 & 0 & 1 & 1 & 3 & 0 & 0 & 0 & 0 & 0 & 0 & 0 & 0 & 0 & 0 & 0 & 0 & 0 & 0 & 0 & 12 \\
\hline & Total 2020 & 325 & 30 & 4 & 0 & 359 & 18 & 0 & 18 & 2 & 38 & 14 & 5 & 0 & 1 & 20 & 4 & 0 & 10 & 0 & 14 & 1 & 1 & 0 & 0 & 1 & 432 \\
\hline
\end{tabular}

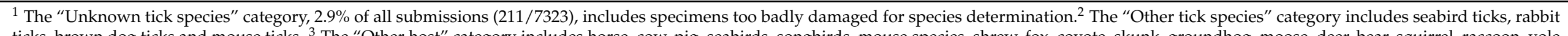

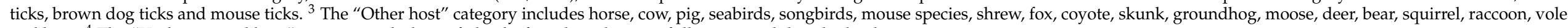
and hare. ${ }^{4}$ The "Undetermined host" category includes unfed ticks found outdoors or fully engorged detached ticks. 
Table 2. Borrelia burgdorferi infection by tick species between 2012 and 2020.

\begin{tabular}{|c|c|c|c|c|c|c|}
\hline \multirow{2}{*}{ Year of Collection } & \multirow{2}{*}{ Collection Location } & \multicolumn{5}{|c|}{ Percentage of Tick Infection (\%) and Samples Size (n) by Tick Species: } \\
\hline & & I. scapularis & I. cookei & D. variabilis & Unknown Species ${ }^{1}$ & Other Species $^{2}$ \\
\hline \multirow{4}{*}{2012} & NB & $8(n=20 / 249)$ & $7(\mathrm{n}=1 / 14)$ & $11(\mathrm{n}=1 / 9)$ & $15(n=4 / 26)$ & $0(\mathrm{n}=0 / 0)$ \\
\hline & NS & $23(\mathrm{n}=9 / 39)$ & $25(\mathrm{n}=1 / 4)$ & $100(\mathrm{n}=1 / 1)$ & $0(\mathrm{n}=0 / 1)$ & $0(\mathrm{n}=0 / 0)$ \\
\hline & PEI & $0(\mathrm{n}=0 / 0)$ & $0(\mathrm{n}=0 / 0)$ & $0(\mathrm{n}=0 / 0)$ & $0(\mathrm{n}=0 / 0)$ & $0(\mathrm{n}=0 / 0)$ \\
\hline & Other $^{3}$ & $33(n=1 / 3)$ & $0(\mathrm{n}=0 / 0)$ & $50(\mathrm{n}=1 / 2)$ & $0(\mathrm{n}=0 / 0)$ & $0(\mathrm{n}=0 / 0)$ \\
\hline \multirow{4}{*}{2013} & NB & $22(\mathrm{n}=71 / 323)$ & $4(n=3 / 84)$ & $0(\mathrm{n}=0 / 35)$ & $0(\mathrm{n}=0 / 9)$ & $0(\mathrm{n}=0 / 1)$ \\
\hline & NS & $14(\mathrm{n}=8 / 58)$ & $0(\mathrm{n}=0 / 2)$ & $0(\mathrm{n}=0 / 15)$ & $0(\mathrm{n}=0 / 2)$ & $0(\mathrm{n}=0 / 0)$ \\
\hline & PEI & $0(\mathrm{n}=0 / 0)$ & $0(\mathrm{n}=0 / 0)$ & $0(\mathrm{n}=0 / 0)$ & $0(\mathrm{n}=0 / 0)$ & $0(\mathrm{n}=0 / 0)$ \\
\hline & Other $^{3}$ & $33(\mathrm{n}=1 / 3)$ & $0(\mathrm{n}=0 / 0)$ & $33(\mathrm{n}=1 / 3)$ & $0(\mathrm{n}=0 / 0)$ & $0(\mathrm{n}=0 / 0)$ \\
\hline \multirow{4}{*}{2014} & NB & $13(\mathrm{n}=67 / 516)$ & $5(n=5 / 109)$ & $7(\mathrm{n}=1 / 14)$ & $9(\mathrm{n}=3 / 34)$ & $0(\mathrm{n}=0 / 0)$ \\
\hline & NS & $17(\mathrm{n}=9 / 53)$ & $13(\mathrm{n}=1 / 8)$ & $0(\mathrm{n}=0 / 9)$ & $0(\mathrm{n}=0 / 2)$ & $0(\mathrm{n}=0 / 0)$ \\
\hline & PEI & $0(\mathrm{n}=0 / 0)$ & $0(\mathrm{n}=0 / 0)$ & $0(\mathrm{n}=0 / 0)$ & $0(\mathrm{n}=0 / 8)$ & $0(\mathrm{n}=0 / 0)$ \\
\hline & Other $^{3}$ & $0(\mathrm{n}=0 / 0)$ & $0(\mathrm{n}=0 / 0)$ & $0(\mathrm{n}=0 / 0)$ & $0(\mathrm{n}=0 / 1)$ & $0(\mathrm{n}=0 / 0)$ \\
\hline \multirow{4}{*}{2015} & NB & $10(\mathrm{n}=47 / 465)$ & $2(\mathrm{n}=2 / 90)$ & $0(\mathrm{n}=0 / 28)$ & $0(\mathrm{n}=0 / 21)$ & $0(\mathrm{n}=0 / 3)$ \\
\hline & NS & $16(n=13 / 82)$ & $0(\mathrm{n}=0 / 7)$ & $0(\mathrm{n}=0 / 60)$ & $0(\mathrm{n}=0 / 0)$ & $0(\mathrm{n}=0 / 1)$ \\
\hline & PEI & $0(\mathrm{n}=0 / 0)$ & $0(\mathrm{n}=0 / 0)$ & $0(\mathrm{n}=0 / 0)$ & $0(\mathrm{n}=0 / 0)$ & $0(\mathrm{n}=0 / 0)$ \\
\hline & Other $^{3}$ & $13(\mathrm{n}=1 / 8)$ & $0(\mathrm{n}=0 / 2)$ & $0(\mathrm{n}=0 / 10)$ & $0(\mathrm{n}=0 / 3)$ & $0(\mathrm{n}=0 / 0)$ \\
\hline \multirow{4}{*}{2016} & NB & $11(\mathrm{n}=75 / 682)$ & $9(\mathrm{n}=7 / 76)$ & $4(n=1 / 24)$ & $5(n=1 / 22)$ & $0(\mathrm{n}=0 / 10)$ \\
\hline & NS & $26(\mathrm{n}=29 / 113)$ & $0(\mathrm{n}=0 / 4)$ & $0(\mathrm{n}=0 / 31)$ & $0(\mathrm{n}=0 / 3)$ & $0(\mathrm{n}=0 / 0)$ \\
\hline & PEI & $33(\mathrm{n}=1 / 3)$ & $0(\mathrm{n}=0 / 0)$ & $0(\mathrm{n}=0 / 0)$ & $0(\mathrm{n}=0 / 1)$ & $0(\mathrm{n}=0 / 0)$ \\
\hline & Other $^{3}$ & $25(n=2 / 8)$ & $0(\mathrm{n}=0 / 0)$ & $0(\mathrm{n}=0 / 16)$ & $0(\mathrm{n}=0 / 14)$ & $0(\mathrm{n}=0 / 1)$ \\
\hline \multirow{4}{*}{2017} & NB & $10(\mathrm{n}=84 / 841)$ & $2(\mathrm{n}=1 / 66)$ & $0(\mathrm{n}=0 / 36)$ & $0(\mathrm{n}=0 / 52)$ & $0(\mathrm{n}=0 / 12)$ \\
\hline & NS & $21(\mathrm{n}=38 / 179)$ & $0(\mathrm{n}=0 / 8)$ & $0(\mathrm{n}=0 / 59)$ & $0(\mathrm{n}=0 / 9)$ & $0(\mathrm{n}=0 / 0)$ \\
\hline & PEI & $0(\mathrm{n}=0 / 1)$ & $0(\mathrm{n}=0 / 0)$ & $0(\mathrm{n}=0 / 0)$ & $0(\mathrm{n}=0 / 0)$ & $0(\mathrm{n}=0 / 0)$ \\
\hline & Other $^{3}$ & $15(\mathrm{n}=6 / 41)$ & $0(\mathrm{n}=0 / 1)$ & $0(\mathrm{n}=0 / 13)$ & $0(\mathrm{n}=0 / 7)$ & $0(\mathrm{n}=0 / 0)$ \\
\hline \multirow{4}{*}{2018} & NB & $22(\mathrm{n}=144 / 519)$ & $5(\mathrm{n}=4 / 88)$ & $5(n=2 / 40)$ & $22(\mathrm{n}=2 / 9)$ & $0(\mathrm{n}=0 / 24)$ \\
\hline & NS & $25(\mathrm{n}=28 / 112)$ & $0(\mathrm{n}=0 / 12)$ & $0(\mathrm{n}=0 / 55)$ & $0(\mathrm{n}=0 / 0)$ & $0(\mathrm{n}=0 / 0)$ \\
\hline & PEI & $25(\mathrm{n}=4 / 16)$ & $0(\mathrm{n}=0 / 0)$ & $0(\mathrm{n}=0 / 2)$ & $0(\mathrm{n}=0 / 0)$ & 100 (n1/1) \\
\hline & Other $^{3}$ & $24(\mathrm{n}=8 / 33)$ & $33(\mathrm{n}=1 / 3)$ & $0(\mathrm{n}=0 / 7)$ & $0(\mathrm{n}=0 / 0)$ & $0(\mathrm{n}=0 / 0)$ \\
\hline \multirow{4}{*}{2019} & NB & $17(\mathrm{n}=103 / 604)$ & $2(\mathrm{n}=2 / 81)$ & $0(\mathrm{n}=0 / 39)$ & $7(\mathrm{n}=1 / 14)$ & $0(\mathrm{n}=0 / 8)$ \\
\hline & NS & $21(\mathrm{n}=31 / 147)$ & $0(\mathrm{n}=0 / 8)$ & $2(n=2 / 89)$ & $0(\mathrm{n}=0 / 7)$ & $0(\mathrm{n}=0 / 0)$ \\
\hline & PEI & $8(n=1 / 13)$ & $0(\mathrm{n}=0 / 0)$ & $0(\mathrm{n}=0 / 1)$ & $0(\mathrm{n}=0 / 1)$ & $0(\mathrm{n}=0 / 0)$ \\
\hline & Other $^{3}$ & $30(\mathrm{n}=6 / 20)$ & $0(\mathrm{n}=0 / 1)$ & $0(\mathrm{n}=0 / 1)$ & $0(\mathrm{n}=0 / 0)$ & $0(\mathrm{n}=0 / 0)$ \\
\hline \multirow{4}{*}{2020} & NB & $18(\mathrm{n}=55 / 307)$ & $5(\mathrm{n}=2 / 37)$ & $15(\mathrm{n}=2 / 13)$ & $19(\mathrm{n}=3 / 16)$ & $0(\mathrm{n}=0 / 1)$ \\
\hline & NS & $18(\mathrm{n}=7 / 40)$ & $0(\mathrm{n}=0 / 0)$ & $0(\mathrm{n}=0 / 5)$ & $0(\mathrm{n}=0 / 0)$ & $0(\mathrm{n}=0 / 0)$ \\
\hline & PEI & $8(\mathrm{n}=2 / 25)$ & $0(\mathrm{n}=0 / 0)$ & $0(\mathrm{n}=0 / 1)$ & $0(\mathrm{n}=0 / 0)$ & $0(\mathrm{n}=0 / 0)$ \\
\hline & Other ${ }^{3}$ & $0(\mathrm{n}=0 / 4)$ & $0(\mathrm{n}=0 / 0)$ & $0(\mathrm{n}=0 / 1)$ & $0(\mathrm{n}=0 / 0)$ & $0(\mathrm{n}=0 / 1)$ \\
\hline
\end{tabular}

${ }^{1}$ The "Unknown tick species" category includes specimens too badly damaged for species determination. ${ }^{2}$ The "Other tick species" category includes seabird ticks, rabbit ticks, brown dog ticks and mouse ticks. " The "Other collection location" category includes ticks from locations other Canadian provinces, the United States, South America, Africa, Asia, Australia and Europe. NA = no counts for that category. Ticks from unknown or multiple possible locations are excluded.

The seasonal distribution of I. scapularis, I. cookei and D. variabilis submissions is shown in Figure 1. The seasonal recovery pattern is similar between collection years (Supplemental Figure S1) and between Maritime provinces (Figure 1). I. scapularis was consistently recovered in all months except February, but most were submitted within two peak periods, the months of April to July and October to November; the spring and fall tick seasons, respectively (Figure 1). The seasonal recovery of ticks from Prince Edward Island has been found to be similar [47]. The seasonal distribution of I. cookei and D. variabilis submissions from New Brunswick and Nova Scotia were also similar between collection years (Supplemental Figure S2); there were insufficient submissions from Prince Edward Island for meaningful comparison. I. cookei was consistently recovered in all months except February and March but, unlike I. scapularis, collection during the fall was modest with most submissions occurring from May to August. D. variabilis donations were received in all months of the year with a peak from May to July but an appreciable number of specimens were collected during the winter months as well. 
A

Proportion of Ixodes scapularis by month from NB, NS and PEI

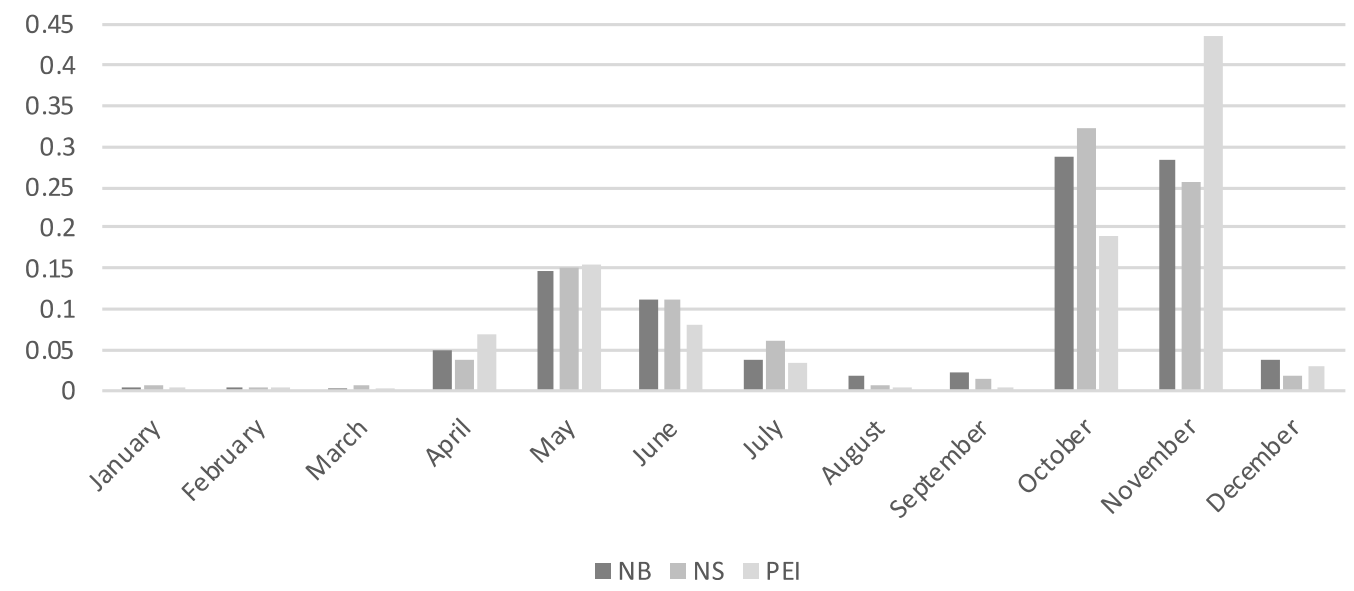

B

Proportion of Ixodes cookei by month from NB ad NS

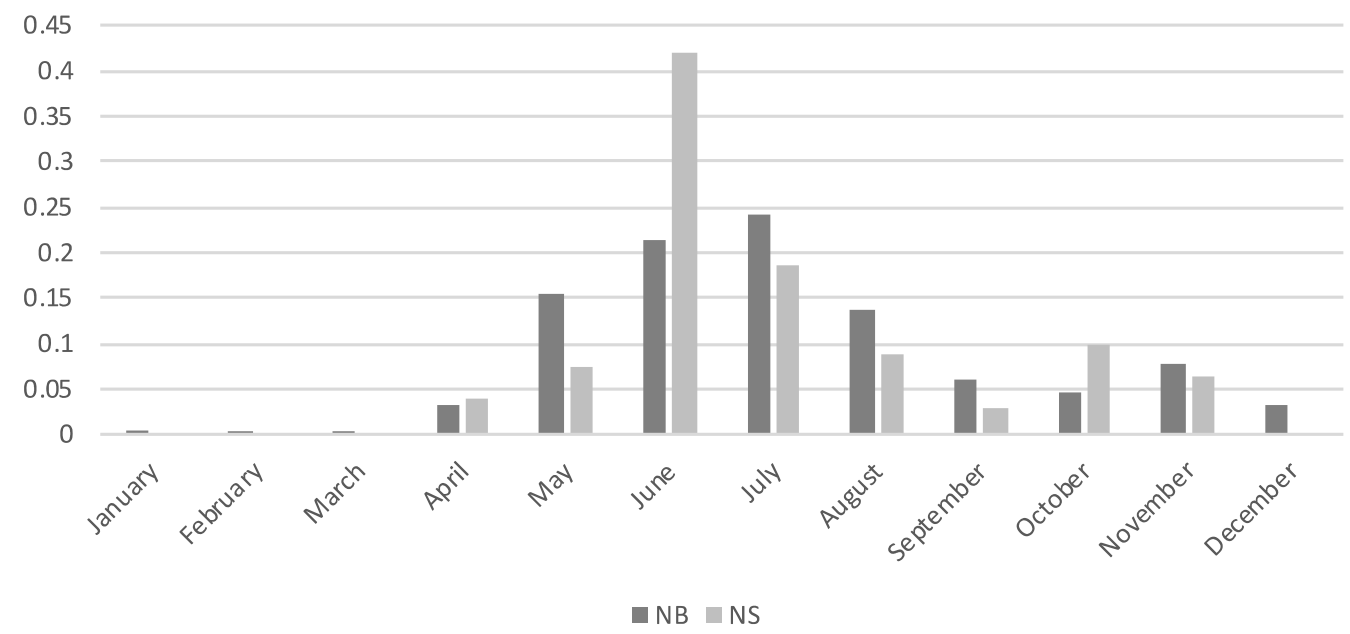

C

Proportion of Dermacentor variabilis by month from NB ad NS

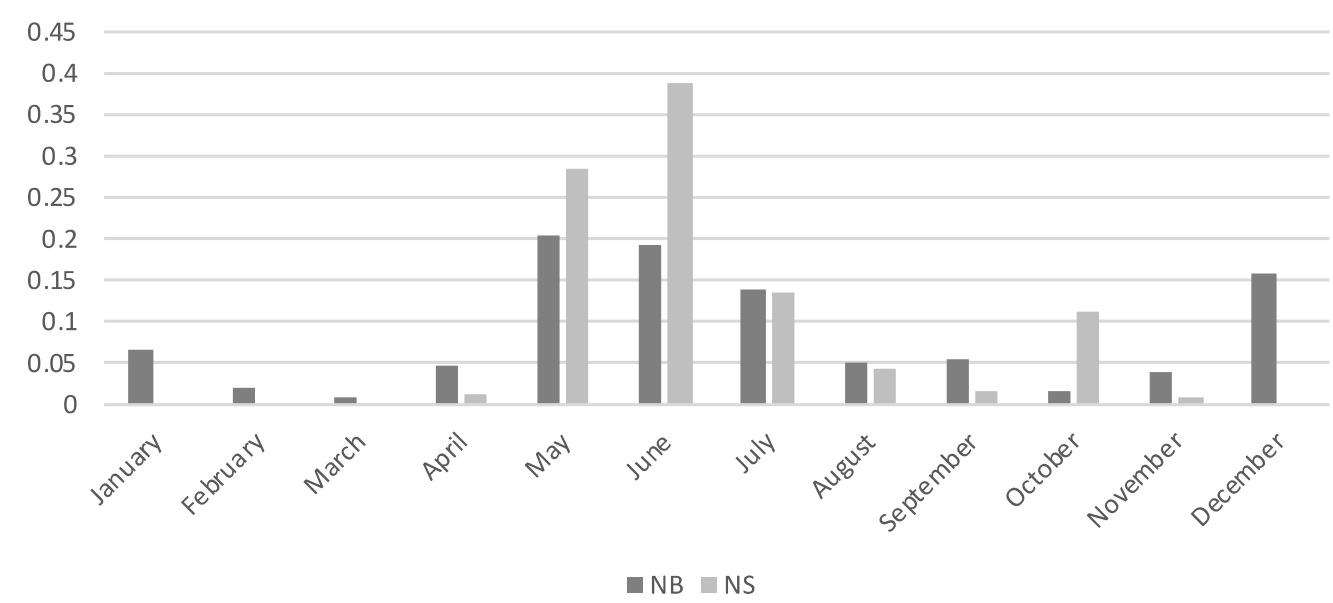

Figure 1. A comparison of seasonal recoveries of Ixodes scapularis (A), Ixodes cookei (B) and Dermacentor variabilis (C) between New Brunswick (NB), Nova Scotia (NS) and Prince Edward Island (PEI). The proportion of the annual tick submissions, per province, is show by month of collection. There were insufficient Ixodes cookei and Dermacentor variabilis recoveries from Prince Edward Island (PEI) for comparison. 


\subsection{Geographic Distribution of I. scapularis Ticks and Borrelia burgdorferi}

This study was promoted throughout New Brunswick so tick recoveries within the province can be assumed to generally reflect tick abundance. In New Brunswick, I. scapularis was collected throughout the province, but mainly from the southern and coastal regions, which include the major population centers of Saint John, Fredericton, and Moncton (Table 3 and Figure 2). At the start of the surveillance period, three clusters of tick recovery were evident in the regions of St. Stephen, Saint John, and Westmorland County (Figure 2). These clusters of increased tick recovery per capita persisted and grew in geographic distribution through the nine-year period. Three major trends were observed over time: growing tick recoveries per capita inland up the Saint John River and beyond, development of growing tick recoveries on a line between Saint John and Westmorland counties and increased recoveries in the north and northeast. Although climate prediction models do not predict that northern New Brunswick would have a climate conducive to tick population establishment [36], when normalized for human population, a key limiting factor in citizen science studies, I. scapularis recoveries from the Miramichi watershed, along the Acadian peninsula and along the Chaleur Bay shows appreciable and increasing numbers of tick recoveries, particularly after 2016 (Figure 2).

Table 3. Ixodes scapularis tick recoveries and percent tick infection from each New Brunswick health region between 2012 and 2020.

\begin{tabular}{|c|c|c|c|c|c|c|c|}
\hline \multirow{2}{*}{ Year of Collection } & \multicolumn{7}{|c|}{ Percentage of Tick, All Species, Infection (\%) and Sample Size (n) by Health Regions: } \\
\hline & Moncton & Saint John & Fredericton & Edmundston & Campbellton & Bathurst & Miramichi \\
\hline 2012 & $9(\mathrm{n}=14 / 157)$ & $5(\mathrm{n}=4 / 87)$ & $33(\mathrm{n}=1 / 3)$ & $0(\mathrm{n}=0 / 0)$ & $0(\mathrm{n}=0 / 0)$ & $0(\mathrm{n}=0 / 2)$ & $0(\mathrm{n}=0 / 0)$ \\
\hline 2013 & $12(\mathrm{n}=10 / 85)$ & $18(\mathrm{n}=16 / 90)$ & $20(\mathrm{n}=2 / 10)$ & $0(\mathrm{n}=0 / 0)$ & $0(\mathrm{n}=0 / 1)$ & $25(\mathrm{n}=1 / 4)$ & $0(\mathrm{n}=0 / 7)$ \\
\hline 2014 & $13(n=23 / 176)$ & $13(n=39 / 299)$ & $16(n=5 / 31)$ & $0(\mathrm{n}=0 / 2)$ & $0(\mathrm{n}=0 / 3)$ & $0(\mathrm{n}=0 / 5)$ & $0(\mathrm{n}=0 / 0)$ \\
\hline 2015 & $6(\mathrm{n}=9 / 149)$ & $10(\mathrm{n}=31 / 307)$ & $8(n=3 / 39)$ & $0(\mathrm{n}=0 / 1)$ & $33(\mathrm{n}=1 / 3)$ & $9(\mathrm{n}=1 / 11)$ & $0(\mathrm{n}=0 / 2)$ \\
\hline 2016 & $12(\mathrm{n}=25 / 208)$ & $11(\mathrm{n}=45 / 410)$ & $11(\mathrm{n}=4 / 36)$ & $0(\mathrm{n}=0 / 1)$ & $0(\mathrm{n}=0 / 4)$ & $0(\mathrm{n}=0 / 15)$ & $0(\mathrm{n}=0 / 12)$ \\
\hline 2017 & $9(n=23 / 256)$ & $11(n=55 / 503)$ & $11(\mathrm{n}=6 / 56)$ & $0(\mathrm{n}=0 / 2)$ & $0(\mathrm{n}=0 / 5)$ & $0(\mathrm{n}=0 / 18)$ & $0(\mathrm{n}=0 / 16)$ \\
\hline 2018 & $12(n=23 / 195)$ & $41(\mathrm{n}=161 / 392)$ & $9(n=4 / 46)$ & $0(\mathrm{n}=0 / 5)$ & $0(\mathrm{n}=0 / 2)$ & $9(\mathrm{n}=4 / 43)$ & $33(\mathrm{n}=6 / 18)$ \\
\hline 2019 & $11(\mathrm{n}=21 / 190)$ & $17(\mathrm{n}=63 / 373)$ & $15(\mathrm{n}=19 / 124)$ & $33(\mathrm{n}=1 / 3)$ & $0(\mathrm{n}=0 / 21)$ & $17(n=5 / 30)$ & $20(\mathrm{n}=1 / 5)$ \\
\hline 2020 & $11(\mathrm{n}=19 / 174)$ & $34(n=53 / 156)$ & $20(\mathrm{n}=7 / 35)$ & $0(\mathrm{n}=0 / 1)$ & $32(n=6 / 19)$ & $40(\mathrm{n}=4 / 10)$ & $0(\mathrm{n}=0 / 1)$ \\
\hline
\end{tabular}

\section{Tick recoveries}

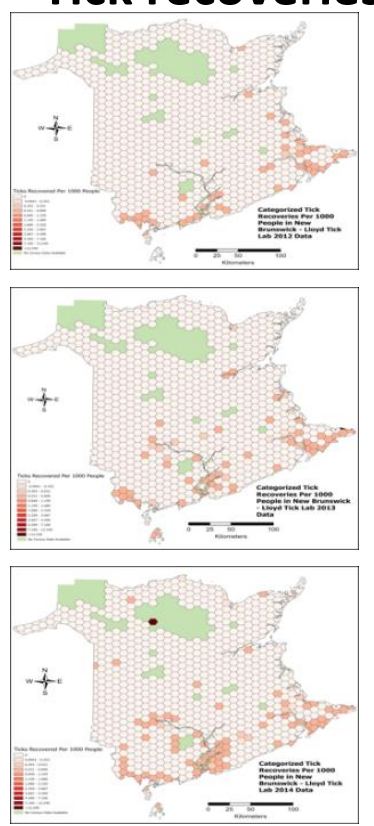

Figure 2. Cont.

\section{Lyme disease Risk}

2012

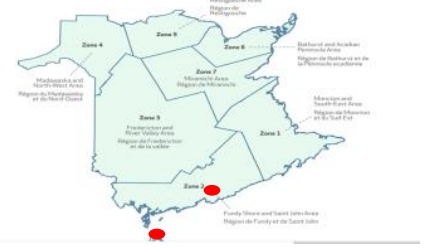

2013

2014

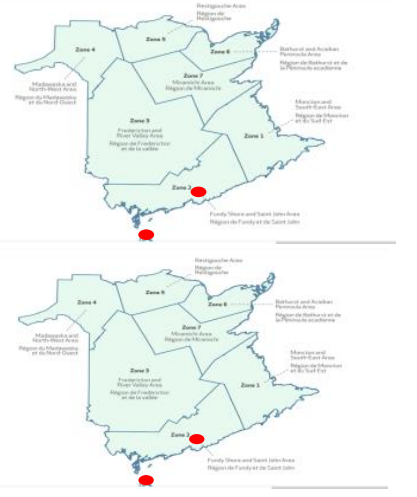



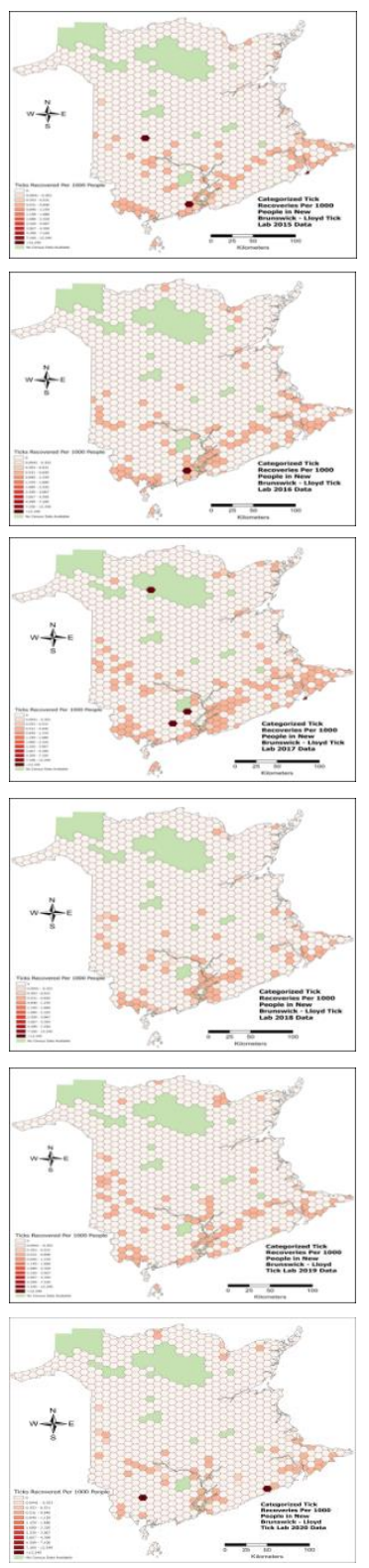

2015

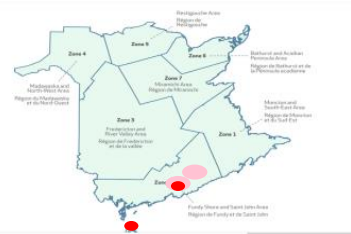

2016

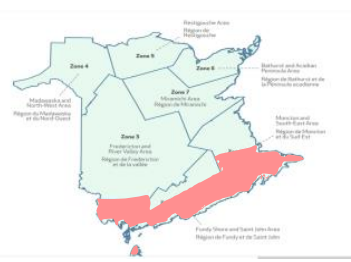

2017

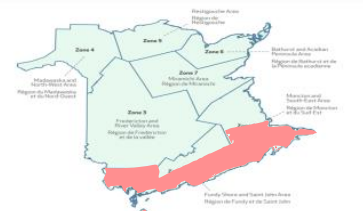

2018

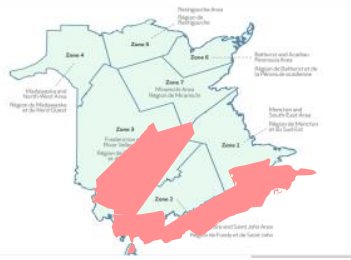

2019

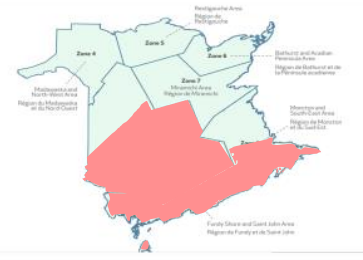

2020

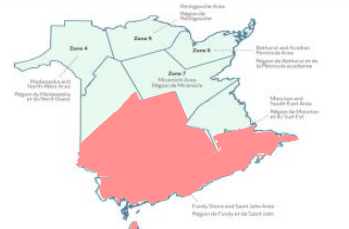

Figure 2. Comparison between tick recovery locations and abundance and public health Ld risk maps for New Brunswick from 2012 to 2020. Images on the left show Ixodes scapularis recoveries mapped using a fine-scale hexagon grid map. Recoveries per 1000 people with a decile colour scale applied to the upper 10th percentile of hexagon-level recovery densities; hexagon cells with no recoveries were grouped into a separate category, those with no census data are shown in green. Images on the right are public health Ld risk regions re-drawn from public health risk maps $[40,41,48]$. In the early maps, red dots indicate known endemic areas, in later years pink indicates broader risk areas. Risk areas may have been added between 2012 and 2015 but maps are not publicly available.

Comparison of tick recovery numbers between provinces is problematic due to different recruitment efforts. Although promoted only in New Brunswick, a substantial number of ticks were submitted from the neighboring provinces of Nova Scotia and Prince Edward Island. In Nova Scotia, ticks were submitted from throughout the mainland, but most were submitted from regions close to New Brunswick, due to the location of the university where the study took place, at the New Brunswick-Nova Scotia border. Fewer ticks were submitted from Prince Edward Island, but Foley-Eby et al. [47] reported ticks encountered throughout the province and in highest numbers in the parts of the province nearest to New Brunswick and Nova Scotia. 
Infection prevalence is not affected by any differential recruitment efforts so it can be compared between provinces (Table 2) and within New Brunswick for which finer-scale geographic tick infection data was collected (Table 3). For all years combined (2012-2020), the province-wide B. burgdorferi infection prevalence in I. scapularis was $14.1 \%$ in New Brunswick (636/4506), and 20.9\% in Nova Scotia (172/832) (Table 2). In this study $9.1 \%$ $(8 / 88)$ of ticks from Prince Edward Island infected which is comparable with the $10.3 \%$ $(37 / 360)$ infection prevalence in I. scapularis collected from Prince Edward Island from 2016-2017 [47]. For New Brunswick, the province-wide infection percentages generally increased over the nine-year study period, whereas in Nova Scotia it remained relatively stable (Table 2). In New Brunswick, all health district regions showed increased tick recoveries and a trend of increased percentages of Borrelia-infected ticks over the course of the study (Table 3, Figure 3). The more northern health districts submitted fewer ticks, but tick recoveries and Borrelia infection prevalence did increase over the course of the study (Table 3, Figure 3).

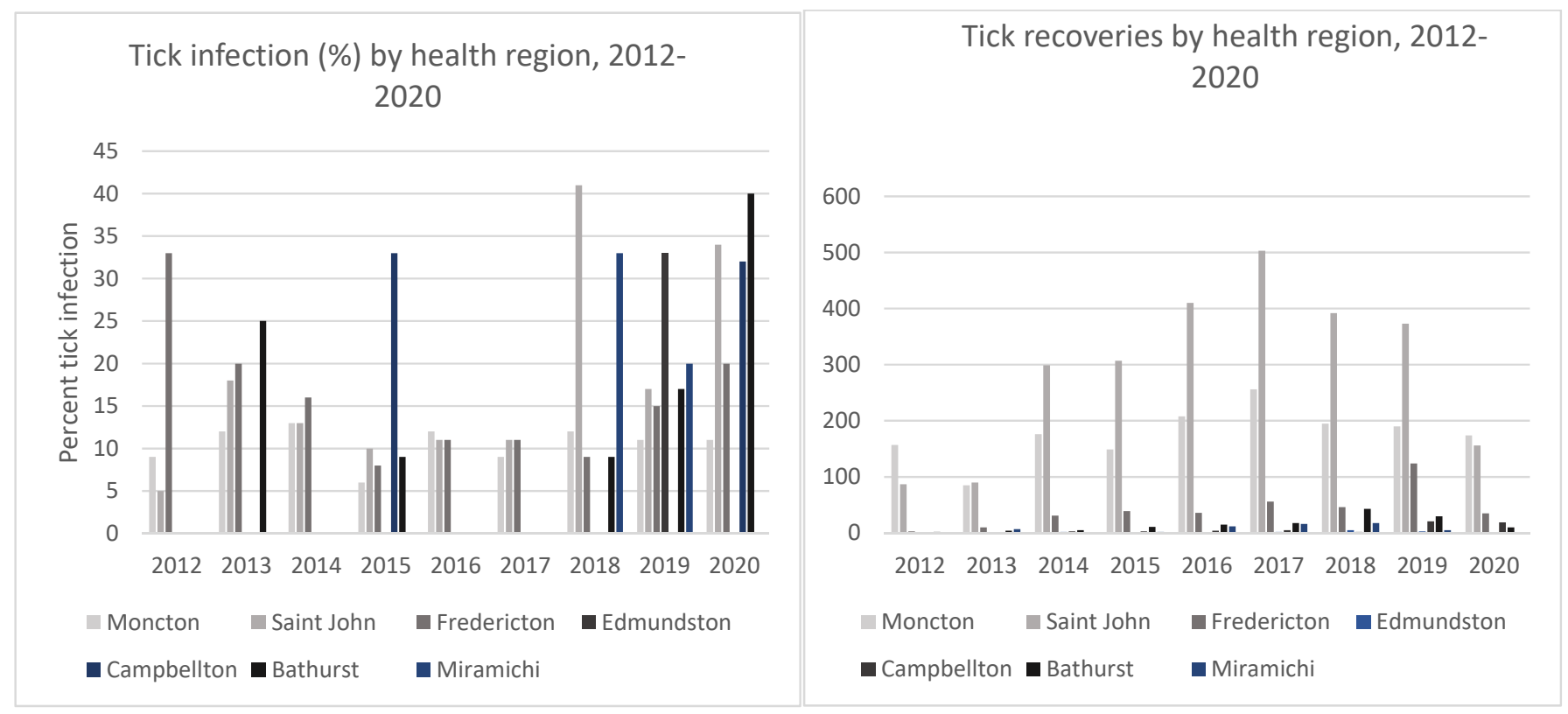

Figure 3. Borrelia burgdorferi infection prevalence in I. scapularis (left) and I. scapularis recoveries (right) across New Brunswick health regions from 2012-2020.

\subsection{Borrelia burgdorferi Infection Prevalence}

All ticks, regardless of species, were tested for the presence of B. burgdorferi (Table 2). The majority of B. burgdorferi-infected ticks were I. scapularis, as expected. Species other than I. scapularis had a low percentage of Borrelia-infected ticks, 3.4\% for I. cookei (24/ 705) and $1.9 \%$ for D. variabilis (12/619) between 2012 and 2020 (Table 2).

The host did not have a significant effect on B. burgdorferi infection of I. scapularis in this collection (Table 4); averaged over the study period, the percent of infection in dogs, cats, humans, other hosts and unknown hosts was $13.3(\mathrm{n}=3622), 15.6(\mathrm{n}=817), 17.9$ $(\mathrm{n}=730), 15.7(\mathrm{n}=41)$ and $22.7(\mathrm{n}=146)$, respectively. The percent of $I$. scapularis infected with $B$. burgdorferi collected from dogs, cats or humans, averaged across years of collection assessed by one-way ANOVA, was not significantly different at $\alpha<0.05$. ( $f$-ratio, 1.40267 , $p=0.265387)$.

The incidence of B. burgdorferi infection in I. scapularis did differ between life stages (Table 5). No larvae were found infected $(\mathrm{n}=33)$, in contrast to adults and nymphs; $B$. burgdorferi was detected in $14.8 \%$ of both tick sex adults $(n=5140)$ and nymphs $(n=215)$. There was no statistically significant difference between infection frequency in adults and nymphs at $\alpha=0.05$ ( $t$-test, averaged across years 2012-2020, $p=1$ ). 
Table 4. Borrelia burgdorferi infection prevalence of Ixodes scapularis by host.

\begin{tabular}{cccccc}
\hline \multirow{2}{*}{ Year of Collection } & \multicolumn{5}{c}{ Percentage of Tick Infection (\%) and Samples Size (n) by Host: } \\
\cline { 2 - 6 } & Dog & Cat & Human & Other Hosts ${ }^{\mathbf{1}}$ & Unknown Hosts $^{2}$ \\
\hline 2012 & $9(\mathrm{n}=16 / 177)$ & $14(\mathrm{n}=10 / 70)$ & $3(\mathrm{n}=1 / 34)$ & $50(\mathrm{n}=1 / 2)$ & $25(\mathrm{n}=2 / 8)$ \\
2013 & $15(\mathrm{n}=26 / 172)$ & $10(\mathrm{n}=3 / 29)$ & $27(\mathrm{n}=7 / 26)$ & $0(\mathrm{n}=0 / 1)$ & $33(\mathrm{n}=1 / 3)$ \\
2014 & $12(\mathrm{n}=50 / 415)$ & $16(\mathrm{n}=10 / 64)$ & $16(\mathrm{n}=4 / 25)$ & $0(\mathrm{n}=0 / 6)$ & $33(\mathrm{n}=2 / 6)$ \\
2015 & $10(\mathrm{n}=42 / 423)$ & $12(\mathrm{n}=11 / 91)$ & $9(\mathrm{n}=4 / 46)$ & $0(\mathrm{n}=0 / 1)$ & $39(\mathrm{n}=5 / 13)$ \\
2016 & $11(\mathrm{n}=61 / 550)$ & $14(\mathrm{n}=20 / 145)$ & $27(\mathrm{n}=25 / 92)$ & $0(\mathrm{n}=0 / 9)$ & $6(\mathrm{n}=1 / 18)$ \\
2017 & $10(\mathrm{n}=67 / 665)$ & $12(\mathrm{n}=19 / 161)$ & $17(\mathrm{n}=36 / 209)$ & $18(\mathrm{n}=2 / 11)$ & $21(\mathrm{n}=9 / 42)$ \\
2018 & $20(\mathrm{n}=85 / 424)$ & $20(\mathrm{n}=23 / 117)$ & $25(\mathrm{n}=32 / 129)$ & $0(\mathrm{n}=0 / 3)$ & $21(\mathrm{n}=3 / 14)$ \\
2019 & $16(\mathrm{n}=81 / 509)$ & $23(\mathrm{n}=26 / 114)$ & $21(\mathrm{n}=24 / 114)$ & $40(\mathrm{n}=2 / 5)$ & $16(\mathrm{n}=5 / 32)$ \\
2020 & $17(\mathrm{n}=49 / 287)$ & $19(\mathrm{n}=5 / 26)$ & $16(\mathrm{n}=9 / 55)$ & $33(\mathrm{n}=1 / 3)$ & $10(\mathrm{n}=1 / 10)$ \\
\hline
\end{tabular}

${ }^{1}$ The "Other hosts" category includes horses, groundhogs, coyotes, moose, deer, bears, cows, skunks, raccoons and rabbits. ${ }^{2}$ The "Unknown hosts" category includes unfed ticks found outdoors not attached to a host or fully engorged detached ticks.

Table 5. Borrelia burgdorferi infection prevalence of Ixodes scapularis by life stage.

\begin{tabular}{|c|c|c|c|c|}
\hline \multirow{2}{*}{ Year of Collection } & \multicolumn{4}{|c|}{ Percentage of Tick Infection (\%) and Samples Size (n) by Developmental Stage: } \\
\hline & Adult & Nymph & Larvae & Unknown \\
\hline 2012 & $11(n=29 / 266)$ & $40(n=2 / 5)$ & $0(\mathrm{n}=0 / 20)$ & $0(\mathrm{n}=0 / 0)$ \\
\hline 2013 & $17(\mathrm{n}=37 / 216)$ & $13(\mathrm{n}=1 / 8)$ & $0(\mathrm{n}=0 / 6)$ & $0(\mathrm{n}=0 / 0)$ \\
\hline 2014 & $13(\mathrm{n}=74 / 569)$ & $8(\mathrm{n}=1 / 12)$ & $0(\mathrm{n}=0 / 0)$ & $0(\mathrm{n}=0 / 0)$ \\
\hline 2015 & $11(\mathrm{n}=58 / 531)$ & $8(n=3 / 37)$ & $0(\mathrm{n}=0 / 4)$ & $0(\mathrm{n}=0 / 3)$ \\
\hline 2016 & $14(\mathrm{n}=112 / 801)$ & $5(\mathrm{n}=1 / 20)$ & $0(\mathrm{n}=0 / 2)$ & $0(\mathrm{n}=0 / 0)$ \\
\hline 2017 & $13(\mathrm{n}=123 / 945)$ & $11(\mathrm{n}=6 / 56)$ & $0(\mathrm{n}=0 / 1)$ & $0(\mathrm{n}=0 / 0)$ \\
\hline 2018 & $20(\mathrm{n}=135 / 675)$ & $20(n=6 / 30)$ & $0(\mathrm{n}=0 / 0)$ & $0(\mathrm{n}=0 / 0)$ \\
\hline 2019 & $17(\mathrm{n}=129 / 760)$ & $28(\mathrm{n}=12 / 43)$ & $0(\mathrm{n}=0 / 0)$ & $0(\mathrm{n}=0 / 0)$ \\
\hline 2020 & $17(\mathrm{n}=64 / 377)$ & $0(\mathrm{n}=0 / 4)$ & $0(\mathrm{n}=0 / 0)$ & $0(\mathrm{n}=0 / 0)$ \\
\hline
\end{tabular}

The incidence of B. burgdorferi infection also did not differ by engorgement status. Averaged across years 2012-2020, B. burgdorferi was detected in $15.0(n=367), 15.1(n=3101)$ and $14.4(n=1958)$ percent of non-engorged, engorged and highly engorged ticks, respectively (Table 6, by one-way ANOVA, $p=0.985543$ ).

Table 6. Borrelia burgdorferi infection prevalence of Ixodes scapularis by engorgement status.

\begin{tabular}{cccc}
\hline \multirow{2}{*}{ Year of Collection } & \multicolumn{2}{c}{ Percentage of Tick Infection (\%) and Sample Size (n) by Engorgement Status:. } \\
\cline { 2 - 4 } & Non-Engorged & Engorged & Highly Engorged \\
\hline 2012 & $8(\mathrm{n}=3 / 36)$ & $11(\mathrm{n}=21 / 193)$ & $8(\mathrm{n}=5 / 63)$ \\
2013 & $40(\mathrm{n}=8 / 20)$ & $11(\mathrm{n}=14 / 130)$ & $19(\mathrm{n}=15 / 80)$ \\
2014 & $4(\mathrm{n}=1 / 26)$ & $14(\mathrm{n}=53 / 380)$ & $7(\mathrm{n}=23 / 176)$ \\
2015 & $10(\mathrm{n}=2 / 21)$ & $13(\mathrm{n}=46 / 354)$ & $14 / 200)$ \\
2016 & $13(\mathrm{n}=6 / 47)$ & $17(\mathrm{n}=65 / 385)$ & $11(\mathrm{n}=49 / 389)$ \\
2017 & $20(\mathrm{n}=13 / 65)$ & $12(\mathrm{n}=73 / 606)$ & $17(\mathrm{n}=44 / 260)$ \\
2018 & $33(\mathrm{n}=1854)$ & $22(\mathrm{n}=81 / 368)$ & $26(\mathrm{n}=60 / 230)$ \\
2019 & $7(\mathrm{n}=5 / 68)$ & $18(\mathrm{n}=86 / 477)$ & $19(\mathrm{n}=27 / 143)$ \\
\hline
\end{tabular}

In New Brunswick, prior to 2015, there were two known tick endemic areas defined by active surveillance (Figure 2). Five suspected areas, all in the Saint John region, had been added by 2015 (Figure 2). The Moncton and Fredericton regions, the two other major population centers in the province, were not considered to be Ld risk areas until 2016 and 2018, respectively. To assess the correlation between tick population densities and $B$. burgdorferi infection prevalence in a region and the designation of that region as a tick endemic/Ld risk areas, tick recoveries and B. burgdorferi infection prevalence were compared between designated endemic/risk areas and those that were not designated 
as endemic/risk areas. One-way ANOVA analysis, showed that I. scapularis recoveries, averaged over the years 2012-2016, which are the years prior to when the risk areas were considered to expand, was indeed significantly different between the regions harboring the three major population centers at $\alpha=0.05$ ( $f$-ratio value $9.10515, p=0.002577)$. A post hoc $t$-test showed that this difference was due to lower tick recoveries from the Fredericton region. However, the difference in recoveries between the St. John region, with known endemic sites, and the Moncton region without known endemic sites, was not significantly different ( $t$-value $-1.5286, p=0.157357$ ) indicating abundant tick recoveries in the Moncton region prior to its designation as a risk area. Because tick infection frequencies are thought to be highest in endemic regions, the non-endemic Moncton and Fredericton regions would be expected to have lower tick infection frequencies, relative to the St. John region with known established tick populations, if they did not harbor endemic regions. However, one-way ANOVA analysis, showed that the percentage of infected I. scapularis from the St. John, Moncton and Fredericton regions, averaged over the years prior to designation of the Moncton and Fredericton regions as risk areas (2012-2016), were not significantly different at $\alpha=0.05$ ( $f$-ratio value $1.81255, p$-value 0.205187 ). This result suggests that despite the apparent great number of ticks encountered and donated in the St. John region, the risk of B. burgdorferi infection existed in the Moncton and Fredericton regions during this time period, prior to their designation as Ld risk areas.

\section{Discussion}

Surveillance of ticks and their pathogens provides information that can aid in the management of tick-vectored diseases in human and veterinary medicine. The importance of such research is emphasized by studies from the United States, Canada and Europe showing that the health costs of Ld, both during and after treatment, are appreciable [49-52]. In this study, we used community-supported passive tick surveillance to monitor the presence of ticks and their Borrelia burgdorferi infection status in Nova Scotia, a province with widely distributed tick populations; New Brunswick, a province with establishing tick populations, and Prince Edward Island, a province thought not to have established tick populations. The community-supported citizen science approach allowed not only extensive tick recoveries over wide geographic areas but also promotes community tick awareness. This study, complemented by a targeted study on Prince Edward Island [47], received abundant tick donations of multiple tick species, from a variety of hosts, from all three Maritime provinces. The tick species most often recovered in this study were the generalist feeders, I. scapularis (Say, 1821), I. cookei (Packard, 1869), and D. variabilis (Say, 1821). These results are consistent with other passive surveillance studies, a review of which is provided in Table 7. B. burgdorferi infection prevalence for I. scapularis in this study was $11.3 \%$ in New Brunswick, $20.9 \%$ in Nova Scotia and Foley-Eby et al. [47] found 10.3\% I. scapularis from Prince Edward Island infected in 2016-2017. The infection prevalence found here for Nova Scotia is slightly higher than reported by Ogden et al. [53] and Dibernardo et al. [44]; however, this work provides a larger sample size with better temporal and geographic resolution.

\subsection{Borrelia burgdorferi and Other Borrelia Species}

Ticks acquire Borrelia burgdorferi infections by feeding from infected hosts. While some Borrelia species such as Borrelia miyamotoi are capable of vertical transmission from an infected female into her eggs, this has not been found for B. burgdorferi, so larvae would not be expected to be infected with B. burgdorferi until they had fed from an infected host [54-56]. This is consistent with our results, although finding of infected larvae is not impossible; larvae can become infected after feeding from an infected host and might be collected prior to molting. B. burgdorferi is efficiently retained during tick molts so nymphs and adults, as well as engorged versus non-engorged ticks, would have additional opportunities to acquire infection with each subsequent blood meal. As a result, in ticks collected from the field and so likely feeding from wildlife reservoirs, one would expect to 
see higher infection prevalence in adults than in the nymphal stage, as well as in engorged vs. non-engorged ticks. In this study, however, ticks were collected from humans and well-cared for companion animals unlikely to be infected prior to the tick feeding so acquiring infection during this terminal feeding is unlikely. This presumably explains the similar infection prevalence of adult and nymphal and engorged versus non-engorged ticks found here.

Each of the major tick species recovered from humans and companion animals in this study can transmit multiple pathogens causing serious disease, although B. burgdorferi, being the most prevalent, is the pathogen of greatest public health concern [1]. The majority of B. burgdorferi-infected ticks found in this study were I. scapularis, as expected given that this species is the primary vector in eastern Canada [6]. However, a low incidence of infection was reproducibly found in ticks of other species; barring the possibility of experimental error, $6 \%$ of I. cookei and $1.9 \%$ of D. variabilis were found to be positive for Borrelia. Ticks acquire B. burgdorferi by feeding, so if these ticks had fed from an infected host, they might become infected. The acquisition of Borrelia by some of the I. cookei and D. variabilis ticks in this study is thus not overly surprising and consistent with previous studies [57-59]. Such infection would not necessarily pose a significant health risk; if the ticks did not efficiently retain the bacteria this would limit transmission to another host. I. cookei is considered to pose much less of a human health risk than I. scapularis for this reason [15]. However, there is a case report of $I$. cookei-transmitted human infection that shows that some risk of transmission exists [14]. An additional layer of complexity is added by the subsequent finding that some of these ticks were hybrids between I. scapularis and I. cookei [60]. The vectoral potential of such hybrids is unknown. In contrast, D. variabilis is considered an incompetent vector as it does not retain Borrelia [58,61-65], however, transmission in rare cases of refeeding cannot be excluded. Both D. variabilis and I. cookei can transmit pathogens other than Borrelia so discounting the health risk posed by these ticks is unwise and, ideally, surveillance should include all tick species that readily feed from humans and the most common pathogens that they transmit.

In addition to the most common species of Borrelia in this region, B. burgdorferi, ticks can carry other Borrelia strains and species. Crowder et al. [66] reported that 39\% of adult $I$. scapularis were infected with at least 2 strains of $B$. burgdorferi sensu stricto. Genospecies other than B. burgdorferi sensu stricto have been reported at a low prevalence in Canada, including B. miyamotoi, B. garinii, and B. bissettii $[44,67]$. Some of the ticks collected from the Canadian Maritime provinces included in this study were infected with $B$. bissettii as well as B. burgdorferi [68] and as the flow of Borrelia genospecies is inter-continental, Eurasian genospecies have and will continue to appear in North America. Infection with different Borrelia species is associated with varying disease manifestations and are poorly or not detected by the two-tiered serological diagnostic algorithm $[69,70]$. Thus, the biodiversity of Borrelia in Canada is of both theoretical and practical importance.

The number of tick donations between provinces can only cautiously be used as a measure of the risk from tick-vectored diseases because of the different recruitment effort in each province. However, the Borrelia infection prevalence is not influenced by recruitment effects, so that metric is a meaningful indication of risk. In Nova Scotia, tick recoveries were robust and the infection prevalence remained around $20 \%$ throughout the study, consistent with the multiple and expanding known established tick populations during this time [39]. The New Brunswick province-wide B. burgdorferi infection prevalence was $8 \%(n=249)$ in 2012. From 2012- 2017, the Borrelia infection prevalence increased to approximately $10 \%$, with the exception of 2013 where it was $22 \%(n=323)$. After 2018, it increased to approximately $20 \%$. Fluctuation in the percentage of ticks infected may be explained by simple annual climactic fluctuations or by sampling error, although recoveries were plentiful. An alternate possibility is that sampling spanned the peak of Borrelia infection that occurs concurrent with tick population establishment. A decrease in Borrelia infection prevalence as reservoir hosts become saturated or resistant coupled with increasing number of ticks collected has been postulated to be an early signal of the transition from adventitious ticks to 
established tick populations [71]. Interestingly, the infection prevalence for Prince Edward Island was also 10\% [47] and has been preceded by steadily increasing tick recoveries and reports of higher tick infection prevalence documented through a collaboration between the veterinary community on the Island and Public Health Agency of Canada researchers [72], raising the possibility that small local tick populations might be established on the Island producing localized cryptic endemic locations.

\subsection{Tick Surveillance}

The risk of a person or animal being infected with Borrelia, or other tick-vectored pathogen, is a function of three events; the chance of encountering a tick, the chance of that tick being infected with Borrelia and the chance of that tick feeding long enough to transmit infection [73]. The number of ticks in a given region can be determined by either active or passive surveillance, with the latter being more sensitive [34]. In the New Brunswick context, this is demonstrated by comparing the results of active and passive tick surveillance. Gabriele-Rivet et al. [74] and Lewis et al. [37] reported recovery of 5 I. scapularis from 159 sites and 9 I. scapularis from 66 sites in 2014, respectively, by active surveillance, whereas the passive surveillance results reported here recovered 744 ticks, 582 of them I. scapularis, that year. Figure 2 shows that the early public health risk maps, based on active surveillance, reflect the relative tick numbers recovered by passive surveillance, but reduced sensitivity of this approach is apparent in the smaller geographic span of risk areas.

Passive tick surveillance has been criticized as being too sensitive as it detects both adventitious ticks and established populations [34]. Although the sensitivity of passive surveillance can be artificially reduced by filtering the data for subsets of ticks such immature stages, males, overwintering adults so that it can match the biological filtering inherent in active tick surveillance, the assumption that adventitious ticks can be disregarded in risk assessment is problematic. While lower in density than ticks from local breeding populations, adventitious ticks do tend to come from established endemic populations, where the chance of a given tick being Borrelia-infected is high [71]. Without Borrelia being established in the local wildlife populations, the initial tick populations seeded from adventitious ticks will be less likely to be infected [71]. However, along migratory routes, episodic introduction of adventitious ticks over time could introduce Borrelia into the wildlife population in advance of the growth of substantial local tick populations. While not surprising in a largely forested and rural province, this suggests that particularly for coastal and inland riparian regions, risk modeling based on tick density alone may be inappropriate. This concern is supported by our finding that the incidence of Borrelia infection in these ticks did not differ significantly between areas within and external to the designated Ld risk areas for several years prior to their designation as Ld risk areas. Ultimately, the preferred method of tick surveillance is one that best captures the risk to people and animals in a region and the passive surveillance tick recovery described here are consistent with canine [75], bovine [76] and equine [77] surveillance initiatives. Canine sentinel studies, that capture the risk from both adventitious ticks and established tick populations, are recognized as important tools in monitoring and predicting regions where individuals are at heightened risk of contracting tick-vectored diseases [78].

\subsection{Lyme Disease Risk Predictions}

For preventative messaging, the risk predictions should either anticipate risk or be concurrent with risk. In this case, tick presence, abundance, the nature and prevalence of infection should be communicated to the public in a timely manner to promote strategies that reduce tick encounters and reduce the duration of tick feeding. While the saltatory and fluid seeding of adventitious ticks is difficult to model, modeling climate and biotic factors that promote establishment of new tick populations can be used to provide predictive power for public health messaging $[36,46,79,80]$. Tick establishment is driven by climate conditions, geography and the movement of animal hosts $[36,46,81-83]$. The similarity 
of the tick recoveries over time and the predicted tick occurrence, as shown in Figure 2, support the predictive power of the occupancy model for New Brunswick, which was constructed using IPCC climate predictions for the 2020's and based on adult tick recoveries from 2014-2016 [36]. Tick populations were found expanding from the south of New Brunswick, along the coastline and upriver valleys in central New Brunswick, as predicted by the model. The upriver increase along the Saint John River and increased spread between Saint John and Westmorland counties are also the areas of highest probability in the 2020's occupancy model [36]. This indicates that forward prediction of trends is accurate, at least in the short term, even at small scale and in complex geographies. However, no climate prediction maps predicted the increased tick recoveries along the northeast coastal regions, showing that tick surveillance is still needed to accurately detect tick presence and the attendant risk of tick-borne disease.

In New Brunswick, a province with establishing tick populations, the public health risk maps based on active tick surveillance have not and do not fully capture tick exposure, as can be seen by comparing actual tick recoveries and public health risk maps (Figure 2). Prior to 2015, risk was indicated as being confined to distinct endemic areas, despite substantial tick recoveries over a much broader area. Results of passive tick surveillance conducted in 2016 by public health agencies, published in June 2017, produced findings similar to those documented here; extensive tick recoveries were obtained in the southern third of province including St. John, Moncton and Fredericton areas [84]. This information may have led to expansion of the Ld risk areas, and generalization of endemic areas to broader "risk areas", which along with the statement that ticks can be encountered anywhere, improves risk messaging to the public. Nevertheless, the officially defined risk areas lag behind substantial tick recoveries by at least 4 years, were infrequently and irregularly updated and still do not encompass coastal areas. New Brunswick is a province with establishing tick populations so the spread of tick populations may be more dynamic than other provinces in which tick populations are either already broadly present, such as Nova Scotia, or thought to be not yet established, as in Prince Edward Island. Nevertheless, endemic areas can expand and the rate of infection increase and adventitious ticks also pose a risk so that timely messaging on tick encounters is necessary to reinforce "tick aware" behaviors regardless of a region's current tick status. Because climate models do not fully capture tick encounters, as we have shown here, tick surveillance will continue to be necessary. This surveillance must be sensitive, which effectively means passive surveillance with public participation, coupled to rapid communication of surveillance results to the public. Annually updated reports from this study have been available online [85,86]. Even more rapid tick encounter information can be provided by online platforms with real time reporting. Such online platforms have shown great success in accomplishing this task in other countries $[87,88]$ and, in Canada, eTick $[89]$ is a dedicated online platform that allows the public to access tick recoveries in real-time.

Table 7. Summary of studies on Ixodes scapularis and Borrelia burgdorferi in Canada, 2000-2016.

\begin{tabular}{|c|c|c|c|c|}
\hline Reference & Province(s) Studied & $\begin{array}{l}\text { Tick Collection } \\
\text { Method(s) }\end{array}$ & $\begin{array}{l}\text { Number of } I . \text { scapularis } \\
\text { Collected }\end{array}$ & $\begin{array}{l}\text { Borrelia Infection Rate } \\
\text { (If Any) }\end{array}$ \\
\hline Banerjee et al., 2000 [43] & ON & $\begin{array}{l}\text { Passive surveillance (dogs) } \\
\text { (1997-1999) }\end{array}$ & 139 & $\begin{array}{l}2 \%(\mathrm{n}=121) \text { by culture } \\
\text { confirmed by monoclonal } \\
\text { antibodies and PCR } \\
6 \%(\mathrm{n}=121) \text { by PCR }\end{array}$ \\
\hline Morshed et al., 2003 [90] & $\mathrm{ON}$ & $\begin{array}{c}\text { Flagging } \\
\text { Trapping small mammals } \\
(1999-2000)\end{array}$ & $\begin{array}{l}263 \\
199\end{array}$ & $\begin{array}{c}14 \% \text { of tick pools }{ }^{1} \\
(\mathrm{n}=86 \text { pools) by PCR } \\
40 \% \text { of white-footed mice } \\
(\mathrm{n}=15) \text { by PCR }\end{array}$ \\
\hline Scott et al., 2004 [91] & ON & $\begin{array}{c}\text { Flagging } \\
\text { Trapping small mammals } \\
(2001-2002)\end{array}$ & $\begin{array}{c}254 \\
59\end{array}$ & $\begin{array}{c}45 \% \text { of cultured pools }{ }^{2} \\
(n=53) \text { by PCR } \\
25 \% \text { of white-footed mice } \\
(n=4) \text { by culturing } \\
\text { confirmed with PCR }\end{array}$ \\
\hline
\end{tabular}


Table 7. Cont

\begin{tabular}{|c|c|c|c|c|}
\hline Reference & Province(s) Studied & $\begin{array}{l}\text { Tick Collection } \\
\text { Method(s) }\end{array}$ & $\begin{array}{l}\text { Number of } I . \text { scapularis } \\
\text { Collected }\end{array}$ & $\begin{array}{l}\text { Borrelia Infection Rate } \\
\text { (If Any) }\end{array}$ \\
\hline Ogden et al., 2006a [53] & $\begin{array}{c}\mathrm{SK}, \mathrm{MB}, \mathrm{ON}, \mathrm{QC}, \mathrm{NB}, \mathrm{NS}, \\
\text { PEI, and NL }\end{array}$ & $\begin{array}{l}\text { Passive surveillance } \\
\quad(1996-2003)\end{array}$ & 1816 & $\begin{array}{c}10 \%(\mathrm{n}=349) \text { in } \mathrm{MB} \\
11 \%(\mathrm{n}=45) \text { in } \mathrm{ON} \\
13 \%(\mathrm{n}=984) \text { in } \mathrm{QC} \\
16 \%(\mathrm{n}=151) \text { in NB} \\
15 \%(\mathrm{n}=86) \text { in NS } \\
11 \%(\mathrm{n}=180) \text { in PE } \\
\mathrm{I} 19 \%(\mathrm{n}=21) \text { in NL } \\
13 \%(\mathrm{n}=1816) \text { in Canada, } \\
\text { all by PCR }\end{array}$ \\
\hline Scott et al., 2007 [92] & $\mathrm{ON}$ & $\begin{array}{c}\text { Flagging } \\
(2005-2006)\end{array}$ & 46 & $\begin{array}{c}\quad 67 \% \text { of pools }{ }^{3} \\
(\mathrm{n}=15 \text { tick pools) by } \\
\text { culture confirmed with } \\
\text { PCR }\end{array}$ \\
\hline Ogden et al., 2008 [24] & $\mathrm{ON}, \mathrm{QC}$, and NS & $\begin{array}{l}\text { Birds capturing } \\
\quad(2005-2006)\end{array}$ & 263 & $\begin{array}{c}15 \%(\mathrm{n}=205 \text { nymphs }) \text { and } \\
0 \%(\mathrm{n}=53 \text { larvae) in ON } \\
\text { No tick was recovered } \\
\text { from QC } \\
25 \% \text { ( } \mathrm{n}=4 \text { nymphs) in NS, } \\
\text { all by PCR }\end{array}$ \\
\hline $\begin{array}{l}\text { Scott and Durden, } 2009 \\
\text { [93] }\end{array}$ & $\mathrm{ON}$ & Bird capturing & 7 & $\begin{array}{l}43 \%(\mathrm{n}=7) \text { by culture and } \\
\text { confirmed with PCR }\end{array}$ \\
\hline Ogden et al., 2010 [71] & QC & $\begin{array}{l}\text { Flagging and rodent } \\
\text { capture (2007-2008) } \\
\text { Passive surveillance } \\
\quad(1996-2004)\end{array}$ & $\begin{array}{c}2259 \text { for active } \\
\text { surveillance } \\
\text { For passive surveillance, } \\
\text { numbers are not given but } \\
\text { submission per year is } \\
\text { shown in their Figure } 1\end{array}$ & $\begin{array}{c}1 \%(\mathrm{n}=1169) \text { by } \\
\text { serological analysis of } \\
\text { rodents } \\
1.8-3.3 \%(\mathrm{n}=675) \text { by PCR } \\
\text { of seropositive rodents } \\
\text { and ticks; } 11 \text { ticks from } \\
1 \text { rodent were pooled }\end{array}$ \\
\hline Bouchard et al., 2011 [94] & QC & $\begin{array}{l}\text { Rodent trapping } \\
\quad(2007-2008)\end{array}$ & 855 & $\begin{array}{l}5 \%(\mathrm{n}=848) \text { of ticks by } \\
\text { PCR } \\
1 \% \text { of rodents were } \\
\text { seropositive }(\mathrm{n}=887) \text { by } \\
\text { immunofluorescence, } \\
\text { ELISA, and Western blot }\end{array}$ \\
\hline Krakowetz et al., 2011 [95] & $\mathrm{MB}, \mathrm{ON}$, and NS & Drag sampling & 153 & $\begin{array}{l}\text { No infection rates } \\
\text { provided }\end{array}$ \\
\hline Dibernardo et al., 2014 [44] & $\begin{array}{c}\mathrm{AB}, \mathrm{MB}, \mathrm{ON}, \mathrm{QC}, \mathrm{NB}, \mathrm{NS}, \\
\text { PEI, and NL }\end{array}$ & Passive surveillance (2012) & 4938 & $\begin{array}{c}14 \%(\mathrm{n}=87) \text { in } \mathrm{AB} \\
9 \%(\mathrm{n}=170) \text { in } \mathrm{MB} \\
16 \%(\mathrm{n}=2591) \text { in } \mathrm{ON} \\
14 \%(\mathrm{n}=1479) \text { in } \mathrm{QC} \\
7 \%(\mathrm{n}=366) \text { in NB} \\
12 \%(\mathrm{n}=34) \text { in NS } \\
10 \%(\mathrm{n}=178) \text { in PEI } \\
27 \%(\mathrm{n}=33) \text { in } \mathrm{NL} \\
\text { All by PCR }\end{array}$ \\
\hline Nelder et al., 2014 [45] & $\mathrm{ON}$ & $\begin{array}{l}\text { Passive surveillance } \\
\quad(2008-2012)\end{array}$ & 7842 & $\begin{array}{c}15 \% \text { of pools } 4 \\
(\mathrm{n}=6046 \text { tick pools }) \text { by } \\
\text { PCR }\end{array}$ \\
\hline Ogden et al., 2014 [96] & $\begin{array}{c}\mathrm{AB}, \mathrm{SK}, \mathrm{MB}, \mathrm{QC}, \mathrm{ON}, \mathrm{NB}, \\
\text { NS, PEI, and NL }\end{array}$ & $\begin{array}{c}\text { Drag sampling } \\
\text { (2008-2013) and trapping } \\
\text { of small mammals } \\
\text { Rodent capturing (2007 } \\
\text { and May-Oct.2008) } \\
\text { Passive surveillance } \\
\text { (2004-2012) }\end{array}$ & $\begin{array}{l}\text { Not provided in the report } \\
\text { but a map of sites where at } \\
\text { least one I. scapularis was } \\
\text { found is given } \\
\text { Rodent results are } \\
\text { presented in the article by } \\
\text { Bouchard et al., } 2011 \\
221\end{array}$ & $\begin{array}{l}\text { No infection rates } \\
\text { provided in this report but } \\
\text { ticks were tested at the } \\
\text { National Microbiology } \\
\text { Laboratory }\end{array}$ \\
\hline Simon et al., 2014 [97] & QC & $\begin{array}{l}\text { Drag sampling } \\
\text { Trapping small mammals }\end{array}$ & $\begin{array}{l}1417 \text { (total for both } \\
\text { collection methods) }\end{array}$ & $\begin{array}{c}14 \%(\mathrm{n}=311) \text { of ticks from } \\
\text { dragging, by PCR } \\
1 \% \text { of ticks from mammals, } \\
\text { by PCR }\end{array}$ \\
\hline
\end{tabular}


Table 7. Cont.

\begin{tabular}{|c|c|c|c|c|}
\hline Reference & Province(s) Studied & $\begin{array}{l}\text { Tick Collection } \\
\text { Method(s) }\end{array}$ & $\begin{array}{l}\text { Number of I. scapularis } \\
\text { Collected }\end{array}$ & $\begin{array}{l}\text { Borrelia Infection Rate } \\
\text { (If Any) }\end{array}$ \\
\hline Werden et al., 2014 [98] & $\mathrm{ON}$ & $\begin{array}{c}\text { Drag sampling } \\
(2009-2010) \\
\text { Trapping small mammals } \\
\text { (2009-2010) }\end{array}$ & $\begin{array}{l}1354 \text { (total for both } \\
\text { collection methods) }\end{array}$ & $\begin{array}{c}\text { Infection rate ranged from } \\
12 \text { to } 30 \%(n=1354) \\
\text { between sites by PCR }\end{array}$ \\
\hline $\begin{array}{c}\text { Gabriele-Rivet et al., } 2015 \\
\text { [74] }\end{array}$ & NB & Drag sampling (2014) & 5 & $25 \%(\mathrm{n}=4)$ by PCR \\
\hline Scott et al., 2016 [99] & $\mathrm{ON}$ & Flagging & 29 & $41 \%(n=29)$ by PCR \\
\hline
\end{tabular}

ON: Ontario, SK: Saskatchewan, MB: Manitoba, QC: Quebec, NB: New Brunswick, NS: Nova Scotia, PEI: Prince Edward Island, NL: Newfoundland and Labrador. ${ }^{1}$ In this study, ticks were tested in pools of up to five adults (normally 3 ) or 28 larvae. ${ }^{2}$ In this study, ticks were tested in pools of up to five adults (normally 3 ) or 7 larvae. ${ }^{3}$ This study did not discuss the pooling arrangement. ${ }^{4}$ Pools from this study groups ticks from the same submission together.

\section{Conclusions}

Ticks are vectors of a number of serious diseases, including $\mathrm{Ld}$. The need for effective and sensitive surveillance needs to be matched by timely communication of risk estimates to the public, recreational and occupational users of outdoor areas, and the veterinary and human healthcare communities. This study provides the largest and highest density information on ticks in the Canadian Maritimes to date and the results have been used to inform residents as well as veterinary and human medical professionals by providing tick identification and infection status of submitted ticks. In addition, the ticks submitted through this study have been used as a national resource for health researchers. This illustrates the intentional integration and respectful partnering with the public and, in particular, those who have increased occupational and recreational exposure to ticks. The results presented here on the value and limitations of tick surveillance demonstrate the need for publicly accessible and integrated information to support tick bite prevention, as well as diagnosis and treatment of tick-vectored diseases.

\section{Materials and Methods}

\subsection{Tick Collection, Identification, and Photography}

Ticks were collected by passive surveillance from veterinarians and the general public across the Maritime provinces between 2012 and 2020 (Animal care protocol 101971). Tick donors filled out a submission form, indicating the tick host, whether tick was attached or not, geographic location of encounter, date of tick collection, and travel within the last 2 weeks, if any. Community-based tick collection method used in this study had the advantages and limitations typically associated with this type of collection process. While efficient, there was a recruitment bias introduced by uneven public awareness of the tick surveillance program. At the initiation of the program, notices were sent to all veterinary clinics across the province of New Brunswick and there was extensive province-wide media coverage. Nevertheless, in the first year of the study the region in the immediate vicinity of the university contributed $12 \%$ of the submitted ticks from New Brunswick, from only 3 veterinary clinics (corresponding to $4 \%$ of the total number of veterinary clinics in the province). By 2017, the Sackville area contributed only $6 \%$ of the total ticks received, suggesting that the regional bias had largely resolved. There was no active promotion of the project in Nova Scotia or Prince Edward Island. However, over the course of the study, awareness of the program expanded, in large part by word of mouth, media coverage, through the lab website and through veterinary networks, subsequently decreasing the extent of the regional bias.

Upon receipt, tick specimens were morphologically identified to species, developmental stage, sex, and engorgement status based on standard keys [100]. Ticks were photographed and laterally bisected, with half archived at $-20{ }^{\circ} \mathrm{C}$ for future study and the other used for molecular testing. Ticks were tested individually and were not pooled. 


\subsection{DNA Extraction}

DNA extraction was performed in a LabGard Class II A2 biological safety cabinet using Aquagenomic solution (MultiTarget Pharmaceuticals) following a modified version of the manufacturer's tissue protocol, as described by Wills et al. [101]. Briefly, ticks were homogenized using an Eppendorf pestle (Diamed) in 50-200 $\mu \mathrm{L}$ of Aquagenomic solution, depending on the tick's size and engorgement. The samples were then incubated in a water bath at $60{ }^{\circ} \mathrm{C}$ for $45 \mathrm{~min}$, vortexed, and centrifuged for four minutes at 13,300 rpm in a desktop microcentrifuge (Spectrafuge 24D Digital Microcentrifuge). The supernatant was transferred into another Eppendorf tube containing 50-200 $\mu \mathrm{L}$ of isopropanol, mixed by inversion, and centrifuged as before. The supernatant was decanted and the DNA pellet rinsed with $50 \mu \mathrm{L}$ of $70 \%$ ethanol. Excess ethanol was pipetted out and the pellet was air-dried for $15 \mathrm{~min}$ at room temperature and $50 \mu \mathrm{L}$ of $1 \mathrm{mM}$ Tris $\mathrm{pH} 7.0$ was added. DNA samples were incubated in a water bath for one hour at $60^{\circ} \mathrm{C}$ to resuspend the DNA, and samples were stored at $-20^{\circ} \mathrm{C}$ for further analysis.

\subsection{Nested Polymerase Chain Reaction}

Nested PCR (nPCR) was performed to detect Borrelia DNA. From 2012-2017, two B. burgdorferi genes, Outer surface protein A (OspA), a plasmid-encoded gene, and Flagellin $B$ (FlagB), a chromosomal gene, were assayed. Borrelia-positive ticks were defined as those with amplification of both Borrelia genes. From 2018 to 2020, the $23 S$ ribosomal RNA ( $r R N A)$ gene was assayed. The outer primers amplify the $23 \mathrm{~S}$ ribosomal RNA (rRNA) gene of all Borrelia spp. [44] and the inner set is specific for B. burgdorferi [102]. Reactions were set up in a PCR cabinet (Misonix) pre-sterilized with UV light and 70\% ethanol. The reaction mixture for the first round consisted of $12.5 \mu \mathrm{L}$ of GoTaq Green Master Mix 2X (Promega), $8.5 \mu \mathrm{L}$ sterile nuclease-free water, $1 \mu \mathrm{L}$ each of $10 \mu \mathrm{M}$ forward and reverse outer primers (Table 8), and $2 \mu \mathrm{L}$ of the extracted DNA or, for negative controls, sterile nuclease-free water. The second round was carried out as the first except for the use of inner primers instead of outer primers (Table 8 ) and $2 \mu \mathrm{L}$ of first round PCR product in lieu of the extracted DNA or water for negative controls. The thermal cycler program was as follows: $95^{\circ} \mathrm{C}$ for five minutes; 40 cycles of $95^{\circ} \mathrm{C}$ for $15 \mathrm{~s}$, annealing temperature for $30 \mathrm{~s}, 72{ }^{\circ} \mathrm{C}$ for $45 \mathrm{~s} ; 72{ }^{\circ} \mathrm{C}$ for five minutes; and hold at $4{ }^{\circ} \mathrm{C}$. The annealing temperature for each primer is listed in Table 8. Amplicons were visualized following electrophoresis in $1.2 \%$ agarose gel in SB buffer $(0.02 \mathrm{M} \mathrm{NaOH}$, $0.075 \mathrm{M}$ boric acid, $\mathrm{pH}$ 8) for one hour at $107 \mathrm{~V}$. Gels were imaged using a BioRad Fluor-STM MultiImager with the Quantity One ${ }^{\circledR} 1$-D analysis software version 4.5.2. While nPCR is considered the most sensitive type of PCR analysis, it is also subject to false positives from contamination [103]. To guard against contamination, all stages of DNA handling (DNA isolation from ticks, PCR reaction preparation, gel electrophoresis) were performed in different rooms with air flows isolated from each other. Additionally, two negative controls were used, one prepared before opening the Eppendorf tube with DNA (before control) and one after (after control). The "before" negative control indicates contamination of reagents while the "after" negative control indicates possible aerosol contamination. Suspect results were discarded and repeated.

\subsection{Geomapping Tick Recoveries}

Tick recoveries from outside of New Brunswick, with uncertain encounter locations or "outside of community" travel history in the 2 weeks prior to recovery were removed. Tick recovery locations were geocoded using an online geocoding resource (https: / www. geocod.io/) to assign a latitude and longitude based on the community in which the tick was found in cases were detailed encounter location was unavailable. Annual tick recovery maps were created using the geographic information systems (GIS) software, ArcGIS, with three layers. The layers were a population census layer obtained from the Center For International Earth Science Information Network-CIESIN-Columbia University, (2018), a point layer that had individual tick recoveries in New Brunswick for the respective year, and a zonal hexagon layer that divided New Brunswick into $\sim 96,010 \mathrm{~km}$ tall, $11.55 \mathrm{~km}$ 
wide hexagons [36]. These layers allowed the tick recovery data to be mapped relative to both location and human per capita values. In citizen science initiatives the human population is a key factor in tick recoveries and the tick recovery data much be corrected for this factor. Some regions encompassing provincial parks, military installations, etc., lacked census data. Across all years, $\sim 90 \%$ of the calculated per capita values resulted in zero or near zero values arising from a low number of ticks found within the respective zone. Therefore, across all years a categorization method was applied that collapsed all zero and near zero values into a single category and expanded the upper $10 \%$ of values for examination and visualization into 11 categories. Therefore, what resulted was a field entitled tickspercapita2012_cat, a categorized field which allowed visual trends to be identified in the tick recoveries per capita throughout the eight-year period.

Table 8. Nested primer sets used to detect Borrelia burgdorferi.

\begin{tabular}{|c|c|c|c|c|c|c|}
\hline Year & Primer Name & $\begin{array}{l}\text { Target } \\
\text { Gene }\end{array}$ & Sequence $\left(5^{\prime}-3^{\prime}\right)$ & $\begin{array}{l}\text { Annealing Temperature } \\
\left({ }^{\circ} \mathrm{C}\right)\end{array}$ & Amplicon Size (bp) & Source \\
\hline \multirow{10}{*}{2012} & OspA out ${ }^{1} \mathrm{R} 1$ & \multirow{3}{*}{ OspA } & GTTAGCAGCCTTGACGAGA & 60 & \multirow{3}{*}{272} & \multirow{3}{*}{$\begin{array}{c}\text { Ogden et al. } 2006 \\
\text { [53] } \\
\text { (OspA1b) } \\
\text { Ogden et al. } 2006 \\
\text { [53] } \\
\text { (OspA4b) }\end{array}$} \\
\hline & & & & & & \\
\hline & OspA out F1 & & GATACTAGTGTTTTGCCATC & & & \\
\hline & OspA in ${ }^{1} \mathrm{R} 1$ & \multirow[b]{2}{*}{ OspA } & GCGTTTCAGTAGATTTGCCTG & 60 & \multirow[b]{2}{*}{214} & \multirow{2}{*}{$\begin{array}{c}\text { Ogden et al. } 2006 \\
\text { [53] } \\
\text { (OspA2b) } \\
\text { Ogden et al. } 2006 \\
\text { [53] } \\
\text { (OspA3b) }\end{array}$} \\
\hline & OspA in F1 & & TCAAGTGTGGTTTGACCTAG & & & \\
\hline & FlagB out R1 & \multirow{3}{*}{ Flag $B$} & AATTGCATACTCAGTACTATTCTTTATAGAT & 60 & \multirow{3}{*}{601} & \multirow{3}{*}{$\begin{array}{c}\text { Ogden et al. } 2006 \\
\text { [53] } \\
\text { (fla outer 2) } \\
\text { Ogden et al. 2006 } \\
\text { [53] } \\
\text { (fla outer 1) }\end{array}$} \\
\hline & & & & & & \\
\hline & FlagB out F1 & & AAGTAGAAAAAGTCTTAGTAAGAATGAAGGA & & & \\
\hline & FlagB in R1 & \multirow[b]{2}{*}{ Flag $B$} & GAAGGTGCTGTAGCAGGTGCTGGCTGT & 60 & \multirow[b]{2}{*}{390} & \multirow{2}{*}{$\begin{array}{c}\text { Ogden et al. } 2006 \\
\text { [53] } \\
\text { (fla inner 2) } \\
\text { Ogden et al. 2006 } \\
\text { [53] } \\
\text { (fla inner 1) }\end{array}$} \\
\hline & FlagB in F1 & & CACATATTCAGATGCAGACAGAGGTTCTA & & & \\
\hline \multirow{6}{*}{2013} & $\begin{array}{l}\text { OspA out R4 } \\
\text { OspA out F4 }\end{array}$ & OspA & $\begin{array}{l}\text { ACAAGAGCAGACGGAACCAG } \\
\text { CCCCTCTAATTTGGTGCCAT }\end{array}$ & 60 & 358 & This work \\
\hline & $\begin{array}{l}\text { OspA in R4 } \\
\text { OspA in F4 }\end{array}$ & OspA & $\begin{array}{l}\text { CACAGGAATTAAAAGCGATGG } \\
\text { AGTGCCTGAATTCCAAGCTG }\end{array}$ & 60 & 220 & This work \\
\hline & $\begin{array}{l}\text { OspA out R3 } \\
\text { OspA out F3 }\end{array}$ & OspA & $\begin{array}{l}\text { GTAATTTCAACTGCTGACCCC } \\
\text { TGAAGGCGTAAAAGCTGAC }\end{array}$ & 60 & 561 & This work \\
\hline & $\begin{array}{l}\text { OspA in R3 } \\
\text { OspA in F3 }\end{array}$ & OspA & $\begin{array}{l}\text { TTGGTGCCATTTGAGTCGTA } \\
\text { ACTTGAATACACAGGAATTA }\end{array}$ & 60 & 330 & This work \\
\hline & $\begin{array}{l}\text { FlagB out } \mathrm{R} 2 \\
\text { FlagB out F2 }\end{array}$ & Flag $B$ & $\begin{array}{l}\text { TGGGGAACTTGATTAGCCTG } \\
\text { TCATTGCCATTGCAGATTGT }\end{array}$ & 60 & 493 & This work \\
\hline & $\begin{array}{l}\text { FlagB in R2 } \\
\text { FlagB in F2 }\end{array}$ & Flag $B$ & $\begin{array}{l}\text { TCATTGCCATTGCAGATTGT } \\
\text { CTTTAAGAGTTCATGTTGGAG }\end{array}$ & 60 & 437 & This work \\
\hline \multirow{4}{*}{2014 to 2017} & $\begin{array}{l}\text { OspA out R2 } \\
\text { OspA out F2 }\end{array}$ & OspA & $\begin{array}{l}\text { CAACTGCTGACCCCTCTAAT } \\
\text { CTTGAAGTTTTCAAAGAAGAT }\end{array}$ & 55 & 487 & This work \\
\hline & $\begin{array}{l}\text { OspA in R2 } \\
\text { OspA in F2 }\end{array}$ & OspA & $\begin{array}{c}\text { TTGGTGCCATTTGAGTCGTA } \\
\text { ACAAGAGCAGACGGAACCAG }\end{array}$ & 58 & 350 & This work \\
\hline & $\begin{array}{l}\text { FlagB out } \mathrm{R} 3 \\
\text { FlagB out F3 }\end{array}$ & FlagB & $\begin{array}{l}\text { GCATCACTTTCAGGGTCTCA } \\
\text { TGGGGAACTTGATTAGCCTG }\end{array}$ & 55 & 503 & This work \\
\hline & $\begin{array}{l}\text { FlagB in R3 } \\
\text { FlagB in F3 }\end{array}$ & Flag $B$ & $\begin{array}{l}\text { CTTTAAGAGTTCATGTTGGAG } \\
\text { TCATTGCCATTGCAGATTGT }\end{array}$ & 58 & 447 & This work \\
\hline 2017 & $\begin{array}{l}\text { OspA out R2 } \\
\text { OspA out F2 }\end{array}$ & OspA & $\begin{array}{l}\text { CAACTGCTGACCCCTCTAAT } \\
\text { CTTGAAGTTTCAAAGAAGAT }\end{array}$ & 55 & 487 & This work \\
\hline \multirow{3}{*}{2018 to 2020} & $\begin{array}{c}23 S \\
\text { out } F \\
23 S \\
\text { out } R\end{array}$ & $\begin{array}{l}\text { Borrelia } \\
\text { spp. } \\
23 S \\
r R N A\end{array}$ & $\begin{array}{l}\text { GTATGTTTAGTGAGGGGGGTG } \\
\text { GGATCATAGCTAGGTGGTTAG }\end{array}$ & 50 & 587 & $\begin{array}{l}\text { Dibernardo et al. } \\
\quad 2014[44]\end{array}$ \\
\hline & \multirow{2}{*}{$\begin{array}{l}23 S \\
\text { in F } \\
23 S \\
\text { in } R\end{array}$} & \multirow{2}{*}{$\begin{array}{c}\text { Borrelia } \\
\text { spp. } \\
23 S \\
r R N A\end{array}$} & ATGTATTCCATTGTTTTAATTACG & 51 & \multirow{2}{*}{340} & \multirow{2}{*}{$\begin{array}{c}\text { Zinck et al. } 2021 \\
{[102]}\end{array}$} \\
\hline & & & GACAAGTATTGTAGCGAGC & & & \\
\hline
\end{tabular}

1 "out" designates the outer nPCR primer sets and "in" designates inner nPCR primer sets. 
Supplementary Materials: The following are available online at https: / www.mdpi.com/article/ 10.3390/pathogens10101284/s1, Figure S1: Seasonal tick recoveries by year. Figure S2: Seasonal recoveries of Ixodes scapularis (A), Ixodes cookei (B) and Dermacentor variabilis (C) in New Brunswick (NB), Nova Scotia (NS) and Prince Edward Island (PEI). The proportion of the annual tick submissions, per province, is show by month of collection. Insufficient Ixodes cookei and Dermacentor variabilis were recovered from Prince Edward Island for comparison.

Author Contributions: Conceptualization V.K.L., D.L.; investigation, J.L., A.M.K., A.F.-E., K.D.H., C.L.F., M.M.; writing—original draft preparation and reviewing, J.L., M.M., D.L.; writing—review and editing, V.K.L., D.L., J.L., A.M.K., C.L.F., A.F.-E., M.M.; supervision, V.K.L. funding acquisition, V.K.L. All authors have read and agreed to the published version of the manuscript.

Funding: This research was funded by the Natural Sciences and Engineering Research Council (NSERC) to VKL and a seed grant by the Canadian Lyme disease Foundation. The mapping component was supported in part by a grant from the Public Health Agency of Canada Climate Change and Infectious Disease Fund.

Acknowledgments: We thank participating veterinary clinics and the private individuals who submitted ticks. We thank the members of the Lloyd lab for discussion.

Conflicts of Interest: The authors declare no conflict of interest. The funders had no role in the design of the study; in the collection, analyses, or interpretation of data; in the writing of the manuscript, or in the decision to publish the results. Subsets of this data have been posted on-line to promote public awareness of tick-vectored diseases at http:/ / www.maritimetickmaps.ca/, accessed on 27 August 2021, and Open Data New Brunswick. Tick Data-2012 to 2018 / Données relatives aux tiques-2012 à 2018-2019 https: / / gnb.socrata.com/Health-and-Wellness/Tick-Data-2012-to-2018 -Donn-es-relatives-aux-tique/3mpw-72pb, accessed on 27 August 2021.

\section{References}

1. Eisen, R.J.; Kugeler, K.J.; Eisen, L.; Beard, C.B.; Paddock, C.D. Tick-borne zoonoses in the United States: Persistent and emerging threats to human health. ILAR J. 2017, 58, 319-335. [CrossRef] [PubMed]

2. Chmelař, J.; Kotál, J.; Karim, S.; Kopacek, P.; Francischetti, I.M.B.; Pedra, J.H.F.; Kotsyfakis, M. Silomes and Mialomes: A system-biology view of tick tissues and tick-host interactions. Trends Parasitol. 2016, 32, 242-254. [CrossRef] [PubMed]

3. Sauer, J.R.; McSwain, J.L.; Bowman, A.S.; Essenberg, R.C. Tick salivary gland physiology. Annu. Rev. Etomol. 1995, 40, 245-267. [CrossRef] [PubMed]

4. Jongejan, F.; Uilenberg, G. The global importance of ticks. Parasitology 2004, 129, S3-S14. [CrossRef] [PubMed]

5. Rudenko, N.; Golovchenko, M.; Grubhoffer, L.; Oliver, J.H., Jr. Updates on Borrelia burgdorferi sensu lato complex with respect to public health. Ticks Tick-Borne Dis. 2011, 2, 123-128. [CrossRef]

6. Sperling, J.L.H.; Sperling, F.A.H. Lyme borreliosis in Canada: Biological diversity and diagnostic complexity from an entomological perspective. Can. Entomol. 2009, 141, 521-549. [CrossRef]

7. Sperling, J.L.H.; Middelveen, M.J.; Klein, D.; Sperling, F.A.H. Evolving perspectives on Lyme borreliosis in Canada. Open Neurol. J. 2012, 6, 94-103. [CrossRef]

8. McCausland, F.R.; Niedermaier, S.; Bijol, V.; Rennke, H.G.; Choi, M.E.; Forman, J.P. Lyme disease-associated glomerulonephritis. Nephrol. Dial. Transplant. 2011, 26, 3054-3056. [CrossRef]

9. Littman, M.P. Lyme nephritis. J. Vet. Emerg. Crit. Care 2013, 23, 163-173. [CrossRef]

10. Bransfield, R.C. Suicide and Lyme and associated diseases. Neuropsychiatr. Dis. Treat. 2017, 13, 1575-1587. [CrossRef]

11. Yeung, C.; Baranchuk, A. Systematic Approach to the Diagnosis and Treatment of Lyme Carditis and High-Degree Atrioventricular Block. Healthcare 2018, 6, 119. [CrossRef]

12. Lindquist, E.; Galloway, T.D.; Artsob, H.; Lindsay, L.R.; Drebot, M.; Wood, H.; Robbins, R.G. A Handbook to the Ticks of Canada (Ixodida: Ixodidae, Argasidae); Biological Survey of Canada: Sackville, NB, Canada, 2016.

13. Piesman, J. Dynamics of Borrelia burgdorferi transmission by nymphal Ixodes dammini ticks. J. Infect. Dis. 1993, 167, 1082-1085. [CrossRef]

14. Hall, J.E.; Amrine, J.W., Jr.; Gais, R.D.; Kolanko, V.P.; Hagenbuch, B.E.; Gerencser, V.F.; Clark, S.M. Parasitization of humans in West Virginia by Ixodes cookei (Acari: Ixodidae), a potential vector of Lyme borreliosis. J. Med. Entomol. 1991, 28, 186-189. [CrossRef]

15. Barker, I.K.; Lindsay, L.R.; Campbell, G.D.; Surgeoner, G.A.; McEwen, S.A. The groundhog tick Ixodes cookei (Acari: Ixodidae): A poor potential vector of Lyme borreliosis. J. Wildl. Dis. 1993, 29, 416-422. [CrossRef]

16. Centers for Disease Control and Prevention. Powassan Virus, 2015a. Available online: http://www.cdc.gov/powassan/ symptoms.html (accessed on 8 September 2016).

17. Centers for Disease Control and Prevention. Rocky Mountain Spotted Fever, 2015b. Available online: https://www.cdc.gov/rmsf/ (accessed on 8 September 2016). 
18. Centers for Disease Control and Prevention. Tularemia, 2015c. Available online: https://www.cdc.gov/tularemia/ signssymptoms /index.html (accessed on 8 September 2016).

19. De la Fuente, J.; Blouin, E.F.; Kocan, K.M. Infection exclusion of the rickettsial pathogen anaplasma marginale in the tick vector Dermacentor variabilis. Clin. Diagn. Lab. Immunol. 2003, 10, 182-184. [CrossRef]

20. Fritzen, C.M.; Huang, J.; Westby, K.; Freye, J.D.; Dunlap, B.; Yabsley, M.J.; Schardein, M.; Dunn, J.R.; Jones, T.F.; Moncayo, A.C. Infection prevalences of common tick-borne pathogens in adult lone star ticks (Amblyomma americanum) and American dog ticks (Dermacentor variabilis) in Kentucky. Am. J. Trop. Med. Hyg. 2011, 85, 718-723. [CrossRef] [PubMed]

21. Dodds, D.G.; Martell, A.M.; Yescott, R.E. Ecology of the American dog tick, Dermacentor variabilis (Say), in Nova Scotia. Can. J. Zool. 1969, 47, 171-181. [CrossRef]

22. Morshed, M.G.; Scott, J.D.; Fernando, K.; Geddes, G.; McNabb, A.; Mak, S.; Durden, L.A. Distribution and characterization of Borrelia burgdorferi isolates from Ixodes scapularis and presence in mammalian hosts in Ontario, Canada. J. Med. Entomol. 2006, 43, 762-773. [CrossRef] [PubMed]

23. Ginsberg, H.S.; Buckley, P.A.; Balmforth, M.G.; Zhioua, E.; Mitra, S.; Buckley, F.G. Reservoir competence of native North American birds for the Lyme disease spirochete, Borrelia burgdorferi. J. Med. Entomol. 2005, 42, 445-449. [CrossRef] [PubMed]

24. Ogden, N.H.; Lindsay, L.R.; Hanincová, K.; Baker, I.K.; Bigras-Poulin, M.; Charron, D.F.; Heagy, A.; Francis, C.M.; O'Callaghan, C.J.; Schwartz, I.; et al. Role of migratory birds in introduction and rage expansion of Ixodes scapularis ticks and of Borrelia burgdorferi and Anaplasma phagocytophilum in Canada. Appl. Environ. Microbiol. 2008, 74, 1780-1790. [CrossRef] [PubMed]

25. Ogden, N.H.; Barker, I.K.; Francis, C.A.; Heagy, A.; Lindsay, L.R.; Hobson, K.A. How far north are migrant birds transporting the tick Ixodes scapularis in Canada? Insights from stable hydrogen isotope analyses of feathers. Ticks Tick-Borne Dis. 2015, 6, 715-720. [CrossRef] [PubMed]

26. Scott, J.D.; Durden, L.A. New record of the Lyme disease bacterium in ticks collected from songbirds in Central and Eastern Canada. Int. J. Acarol. 2015, 41, 241-249. [CrossRef]

27. Scott, J.D. Studies abound on how far north Ixodes scapularis ticks are transported by birds. Tick Tick-Borne Dis. 2016, 7, 327-328. [CrossRef] [PubMed]

28. Neelakanta, G.; Sultana, H.; Fish, D.; Anderson, J.F.; Fikrig, E. Anaplasma phagocytophilum induces Ixodes scapularis ticks to express and antifreeze glycoprotein gene that enhances their survival in the cold. J. Clin. Invest. 2010, 120, 3179-3190. [CrossRef]

29. Talbot, B.; Slatculescu, A.; Thickstun, C.R.; Koffi, J.K.; Leighton, P.A.; McKay, R.; Kulkarni, M.A. Landscape determinants of density of blacklegged ticks, vectors of Lyme disease, at the northern edge of their distribution in Canada. Sci. Rep. 2019, 9, 16652. [CrossRef] [PubMed]

30. Dantas-Torres, F. Climate change, biodiversity, ticks and tick-borne diseases: The butterfly effect. Int. J. Parasitol. Parasites Wildl. 2015, 28, 452-461. [CrossRef] [PubMed]

31. Waddell, L.A.; Greig, J.; Lindsay, L.R.; Hinckley, A.F.; Ogden, N.H. A systematic review on the impact of gestational Lyme disease in humans on the fetus and newborn. PLOS ONE 2018, 13, e0207067. [CrossRef]

32. Public Health Agency of Canada. Surveillance of Lyme Disease. Available online: http://healthycanadians.gc.ca/diseasesconditions-maladies-affections/disease-maladie/lyme/surveillance-eng.php (accessed on 1 December 2020).

33. Leeflang, M.M.; Ang, C.W.; Berkhout, J.; Bijlmer, H.A.; Van Bortel, W.; Brandenburg, A.H.; Van Burgel, N.D.; Van Dam, A.P.; Dessau, R.B.; Fingerle, V.; et al. The diagnostic accuracy of serological tests for Lyme borreliosis in Europe: A systematic review and meta-analysis. BMC Infect. Dis. 2016, 16, 140. [CrossRef]

34. Koffi, J.K.; Leighton, P.A.; Pelcat, Y.; Trudel, L.; Lindsay, L.R.; Milord, F.; Ogden, N.H. Passive surveillance for I. scapularis ticks: Enhanced analysis for early detection of emerging Lyme disease risk. J. Med. Entomol. 2012, 49, 400-409. [CrossRef]

35. Gherman, C.M.; Mihalca, A.D.; Dumitrache, M.O.; Gyoörke, A.; Oroian, I.; Sandor, M.; Cozma, V. CO 2 flagging—An improved method for the collection of questing ticks. Parasites Vectors 2012, 5, 125. [CrossRef]

36. Lieske, D.J.; Lloyd, V.K. Combining public participatory surveillance and occupancy modelling to predict the distributional response of Ixodes scapularis to climate change. Ticks Tick-Borne Dis. 2018, 9, 695-706. [CrossRef] [PubMed]

37. Lewis, J.; Boudreau, C.R.; Patterson, J.W.; Bradet-Legris, J.; Lloyd, V.K. Citizen science and community engagement in tick surveillance-A Canadian case study. Healthcare 2018, 6, 22. [CrossRef] [PubMed]

38. Ogden, N.H.; Lindsay, L.R.; Morshed, M.; Sockett, P.N.; Artsob, H.A. The emergence of Lyme disease in Canada. Can. Med. Assoc. J. 2009, 180, 1221-1224. [CrossRef] [PubMed]

39. Nova Scotia. Communicable Disease and Prevention Control; Nova Scotia: Halifax, NS, Canada, 2013.

40. New Brunswick Disease Watch Bulletin. 2015. Available online: http://www2.gnb.ca/content/dam/gnb/Departments/h-s/ pdf/en/Publications/NBDiseaseWatchBulletin_vol21.pdf (accessed on 3 December 2020).

41. New Brunswick Office of the Chief Medical Officer of Health (Public Health). Lyme Disease-Brief Reference for New Brunswick Clinicians. Available online: https://www2.gnb.ca/content/gnb/en/departments/ocmoh/cdc/content/vectorborne_ andzoonotic/Tick-Borne_Diseases/brief.html (accessed on 3 December 2020).

42. Cawthorn, R.J.; Horney, B.S.; Maloney, R. Lyme disease vector, Ixodes dammini (the northern deer tick), identified in Prince Edward Island. Can. Vet. J. 1990, 31, 220.

43. Banerjee, S.N.; Banerjee, M.; Fernando, K.; Scott, J.D.; Mann, R.; Morshed, M.G. Presence of spirochete causing Lyme disease, Borrelia burgdorferi, in the blacklegged tick, Ixodes scapularis, in Southern Ontario. Can. Med. Assoc. J. 2000, 162, 1567-1569. 
44. Dibernardo, A.; Cote, T.; Ogden, N.H.; Lindsay, L.R. The prevalence of Borrelia miyamotoi infection, and co-infections with other Borrelia spp. in Ixodes scapularis ticks collected in Canada. Parasites Vectors 2014, 7, 183. [CrossRef]

45. Nelder, M.P.; Russell, C.; Lindsay, L.R.; Dhar, B.; Patel, S.N.; Johnson, S.; Moore, S.; Kristjanson, E.; Li, Y.; Ralevski, F. Populationbased passive tick surveillance and detection of expanding foci of blacklegged ticks Ixodes scapularis and the Lyme disease agent Borrelia burgdorferi in Ontario, Canada. PLoS ONE 2014, 9, e105358. [CrossRef]

46. Ogden, N.H.; Bigras-Poulin, M.; O'Callaghan, C.J.; Barker, I.K.; Lindsay, L.R.; Maarouf, A.; Smoyer-Tomic, K.E.; Waltner-Toews, D.; Charron, D. A dynamic population model to investigate effects of climate on geographic range and seasonality of the tick Ixodes scapularis. Int. J. Parasitol. 2006, 35, 375-389. [CrossRef]

47. Foley-Eby, A.H.; Savidge, C.; Lloyd, V.K. Ixodes scapularis ticks and Borrelia burgdorferi on Prince Edward Island: Passive tick surveillance and canine seroprevalence. Can. Vet. J. 2020, 61, 1107-1110.

48. Global News, 2017: New Blacklegged Tick Risk Areas Identified in New Brunswick. Available online: https://globalnews.ca/ news/3762878/new-tick-risk-areas-new-brunswick/ (accessed on 9 December 2020).

49. Adrion, E.R.; Aucott, J.; Lemke, K.W.; Weiner, J.P. Health care costs, utilization and patterns of care following Lyme disease. PLoS ONE 2015, 10, e0116767. [CrossRef]

50. Van den Wijngaard, C.C.; Hofhuis, A.; Wong, A.; Harms, M.G.; de Wit, G.A.; Lugner, A.K.; Suijkerbuijk, A.W.M.; Mangen, M.-J.J.; van Pelt, W. The cost of Lyme borreliosis. Eur. J. Public Health 2017, 27, 538-547. [CrossRef]

51. Davidsson, M. The Financial Implications of a Well-Hidden and Ignored Chronic Lyme Disease Pandemic. Healthcare 2018, 6, 16. [CrossRef] [PubMed]

52. Mac, S.; da Silva, S.R.; Sander, B. The economic burden of Lyme disease and the cost-effectiveness of Lyme disease interventions: A scoping review. PLoS ONE 2019, 14, e0210280. [CrossRef] [PubMed]

53. Ogden, N.H.; Trudel, L.; Artsob, H.; Barker, I.K.; Beauchamp, G.; Charron, D.F.; Drebot, M.A.; Galloway, T.D.; O’Handley, R.; Thompson, R.A.; et al. Ixodes scapularis ticks collected by passive surveillance in Canada: Analysis of geographic distribution and infection with Lyme borreliosis agent Borrelia burgdorferi. J. Med. Entomol. 2006, 43, 600-609. [CrossRef] [PubMed]

54. Scoles, G.A.; Papero, M.; Beati, L.; Fish, D. A relapsing fever group spirochete transmitted by Ixodes scapularis ticks. Vector Borne Zoonotic Dis. 2001, 1, 21-34. [CrossRef] [PubMed]

55. Rollend, L.; Fish, D.; Childs, J.E. Transovarial transmission of Borrelia spirochetes by Ixodes scapularis: A summary of the literature and recent observations. Ticks Tick-Borne Dis. 2013, 4, 46-51. [CrossRef]

56. Han, S.; Lubelczyk, C.; Hickling, G.J.; Belperron, A.A.; Bockenstedt, L.K.; Tsao, J.I. Vertical transmission rates of Borrelia miyamotoi in Ixodes scapularis collected from white-tailed deer. Ticks Tick Borne Dis. 2019, 10, 682-689. [CrossRef] [PubMed]

57. Anderson, J.F.; Johnson, R.C.; Magnarelli, L.A.; Hyde, F.W. Identification of endemic foci of Lyme disease: Isolation of Borrelia burgdorferi from feral rodents and ticks (Dermacentor variabilis). J. Clin. Microbiol. 1985, 22, 36-38. [CrossRef] [PubMed]

58. Lindsay, L.R.; Barker, I.K.; Surgeoner, G.A.; McEwen, S.A.; Elliott, L.A.; Kolar, J. Apparent incompetence of Dermacentor variabilis (Acari: Ixodidae) and fleas (Insecta: Siphonaptera) as vectors of Borrelia burgdorferi in an Ixodes scapularis endemic area of Ontario, Canada. J. Med. Entomol. 1991, 28, 750-753. [CrossRef]

59. Scott, J.; Clark, K.L.; Anderson, J.F.; Foley, J.E.; Young, M.R.; Durden, L.A. Lyme Disease Bacterium, Borrelia burgdorferi sensu lato, Detected in Multiple Tick Species at Kenora, Ontario, Canada. J. Bacteriol. Parasitol. 2017, 8, 1. [CrossRef]

60. Patterson, J.W.; Duncan, A.M.; McIntyre, K.C.; Lloyd, V.K. Evidence for genetic hybridization between Ixodes scapularis and Ixodes cookei. Can. J. Zool. 2017, 95, 527-537. [CrossRef]

61. Piesman, J.; Sinsky, R.J. Ability of Ixodes scapularis, Dermacentor variabilis, and Amblyomma americanum (Acari: Ixodidae) to acquire, maintain, and transmit Lyme disease spirochete (Borrelia burgdorferi). J. Med. Entomol. 1988, 25, 336-338. [CrossRef]

62. Sanders, F.H., Jr.; Oliver, J.H., Jr. Evaluation of Ixodes scapularis, Amblyomma americanum, and Dermacentor variabilis (Acari: Ixodidae) from Georgia as vectors of a Florida strain of the Lyme disease spirochete, Borrelia burgdorferi. J. Med. Entomol. 1995, 32, 402-406. [CrossRef] [PubMed]

63. Kocan, A.A.; Mukolwe, S.W.; Murphy, G.L.; Barker, R.W.; Kocan, K.M. Isolation of Borrelia burgdorferi (Spirochaetales: Spirochaetaceae) from Ixodes scapularis and Dermacentoralbipictus ticks (Acari: Ixodidae) in Oklahoma. J. Med. Entomol. 1992, 29, 630-633. [CrossRef]

64. Johns, R.; Sonenshine, D.E.; Hynes, W.L. Identification of a defensin from the hemolymph of the American dog tick, Dermacentor variabilis. Insect Biochem. Mol. Biol. 2001, 31, 857-865. [CrossRef]

65. Johns, R.; Ohnishi, J.; Broadwater, A.; Sonenshine, D.E.; De Silva, A.M.; Hynes, W.L. Contrasts in tick innate immune responses to Borrelia burgdorferi challenge: Immunotolerance in Ixodes scapularis versus immunocompetence in Dermacentor variabilis (Acari: Ixodidae). J. Med. Entomol. 2001, 38, 99-107. [CrossRef] [PubMed]

66. Crowder, C.D.; Matthews, H.E.; Schutzer, S.; Rounds, M.A.; Luft, B.J.; Nolte, O.; Campbell, S.R.; Phillipson, C.A.; Li, F.; Sampath, R.; et al. Genotypic variation and mixtures of Lyme Borrelia in Ixodes ticks from North America and Europe. PLoS ONE 2010, 5, e10650. [CrossRef]

67. Smith, R.P.; Muzaffar, S.B.; Lavers, J.; Lacombe, E.H.; Cahill, B.K.; Lubelczyk, C.B.; Kinsler, A.; Mathers, A.J.; Rand, P.W. Borrelia garinii in seabird ticks (Ixodes uriae), Atlantic coast, North America. Emerg. Infect. Dis. 2006, 12, 1909-1911. [CrossRef]

68. Lewis, J.; Lloyd, V.K. Identification of Borrelia bissettii in Ixodes scapularis ticks from New Brunswick, Canada. Can. J. Microbiol. 2019, 65, 155-161. [CrossRef]

69. Pachner, A.R.; Dail, D.; Bai, Y.; Sondey, M.; Pak, L.; Narayan, K.; Cadavid, D. Genotype determines phenotype in experimental Lyme borreliosis. Ann. Neurol. 2004, 56, 361-370. [CrossRef] 
70. Stanek, G.; Reiter, M. The expanding Lyme Borrelia complex-Clinical significance of genomic species? Clin. Microbiol. Infect. 2011, 17, 487-493. [CrossRef]

71. Ogden, N.H.; Bouchard, C.; Kurtenbach, K.; Margos, G.; Lindsay, L.R.; Trudel, L.; Nguon, S.; Milord, F. Active and passive surveillance and phylogenetic analysis of Borrelia burgdorferi elucidate the process of Lyme disease risk emergence in Canada. Environ. Health Perspect. 2010, 118, 909-914. [CrossRef]

72. Quigley, R. Little local worry about tick populations. J. Pioneer 2011. Available online: https://www.journalpioneer.com/news/ local/little-local-worry-about-tick-populations-52682/ (accessed on 11 December 2020).

73. Lloyd, V.K.; Hawkins, R.G. Under-Detection of Lyme Disease in Canada. Healthcare 2018, 6, 125. [CrossRef]

74. Gabriele-Rivet, V.; Arsenault, J.; Badcock, J.; Cheng, A.; Edsall, J.; Goltz, J.; Kennedy, J.; Lindsay, L.R.; Pelcat, Y.; Ogden, N.H. Different ecological niches for ticks of public health significance in Canada. PLoS ONE 2015, 10, e0131282. [CrossRef]

75. Bjurman, N.K.; Bradet, G.; Lloyd, V.K. Assessing the risk of Borrelia infection in New Brunswick: Using dogs as a sentinel species. Can. Vet. J. 2016, 57, 1-4.

76. McGowan, C.V. The Seroprevalence of Borrelia burgdorferi in New Brunswick and Nova Scotia Cows (Bos taurus) and Transmission of Borrelia to Unpasteurized Milk. B.Sc. Honours Thesis, Mount Allison University, Sackville, NB, Canada, 2019.

77. Bush, E. Borrelia Infection in Maritime Horses, 2018. B.Sc. Honours Thesis, Mount Allison University, Sackville, NB, Canada, 2018.

78. Lindenmayer, J.M.; Marshall, D.; Onderdonk, A.B. Dogs as sentinels for Lyme disease in Massachusetts. Am. J. Public Health 1991, 81, 1448-1455. [CrossRef] [PubMed]

79. Soucy, J.R.; Slatculescu, A.M.; Nyiraneza, C.; Ogden, N.H.; Leighton, P.A.; Kerr, J.T.; Kulkarni, M.A. High-Resolution Ecological Niche Modeling of Ixodes scapularis Ticks Based on Passive Surveillance Data at the Northern Frontier of Lyme Disease Emergence in North America. Vector-Borne Zoonotic Dis. 2018, 18, 235-242. [CrossRef] [PubMed]

80. Slatculescu, A.M.; Clow, K.M.; McKay, R.; Talbot, B.; Logan, J.J.; Thickstun, C.R.; Jardine, C.M.; Ogden, N.H.; Knudby, A.J.; Kulkarni, M.A. Species distribution models for the eastern blacklegged tick, Ixodes scapularis, and the Lyme disease pathogen, Borrelia burgdorferi, in Ontario, Canada. PLoS ONE 2020, 15, e0238126. [CrossRef] [PubMed]

81. Kotchi, S.O.; Bouchard, C.; Brazeau, S.; Ogden, N.H. Earth Observation-Informed Risk Maps of the Lyme Disease Vector Ixodes scapularis in Central and Eastern Canada. Remote Sens. 2021, 13, 524. [CrossRef]

82. Ogden, N.H.; Maarouf, A.; Barker, I.K.; Bigras-Poulin, M.; Lindsay, L.R.; Morshed, M.G.; O'Callaghan, C.J.; Ramay, F.; WaltnerToew, D.; Charron, D.F. Climate change and the potential for range expansion of the Lyme disease vector Ixodes scapularis in Canada. Int. J. Parasitol. 2006, 36, 63-70. [CrossRef]

83. Ogden, N.H.; Radojević, M.; Wu, X.; Duvvuri, V.R.; Leighton, P.A.; Wu, J. Estimated effects of projected climate change on the basic reproductive number of the Lyme disease vector Ixodes scapularis. Environ. Health Perspect. 2014, 122, 631-638. [CrossRef] [PubMed]

84. Disease Watch 26: 06/17. 2017. Available online: https://www2.gnb.ca/content/gnb/en/departments/ocmoh/publications. html (accessed on 21 September 2021).

85. Maritime Tick Information Portal. Available online: http://www.maritimetickmaps.ca/ (accessed on 21 September 2021).

86. Open Data New Brunswick. Tick Data-2012 to 2018/Données Relatives Aux Tiques—2012 à 2018-Tick Timeline. 2019. Available online: https://gnb.socrata.com/Health-and-Wellness/Tick-Data-2012-to-2018-Donn-es-relatives-aux-tique/3mpw72pb.TickTimeline_Chronologiedetiques2012-2018.mp4 (accessed on 8 December 2020).

87. Kopsco, H.L.; Duhaime, R.J.; Mather, T.N.; Diuk-Wasser, M. Crowdsourced Tick Image-Informed Updates to U.S. County Records of Three Medically Important Tick Species. J. Med. Entomol. 2021, tjab082. [CrossRef] [PubMed]

88. Cull, B. Potential for online crowdsourced biological recording data to complement surveillance for arthropod vectors. PLoS ONE 2021, 16, e0250382. [CrossRef] [PubMed]

89. Koffi, J.; Savage, J.; Thivierge, K.; Lindsay, L.R.; Bouchard, C.; Pelcat, Y.N.; Ogden, N. Evaluating the submission of digital images as a method of surveillance for Ixodes scapularis ticks. Parasitology 2017, 144, 877-883. [CrossRef] [PubMed]

90. Morshed, M.G.; Scott, J.D.; Fernando, K.; Mann, R.B.; Durden, L.A. Lyme disease spirochete, Borrelia burgdorferi endemic at epicenter in Rondeau Provincial Park, Ontario. J. Med. Entomol. 2003, 40, 91-94. [CrossRef]

91. Scott, J.D.; Fernando, K.; Durden, L.A.; Morshed, M.G. Lyme disease spirochete, Borrelia burgdorferi, endemic in epicenter at Turkey Point, Ontario. J. Med. Entomol. 2004, 41, 226-230. [CrossRef] [PubMed]

92. Scott, J.D.; Lee, M.-K.; Fernando, K.; Jorgensen, D.R.; Durden, L.A.; Morshed, M.G. Rapid introduction of Lyme disease spirochete, Borrelia burgdorferi sensu stricto, in Ixodes scapularis (Acari: Ixodidae) established at Turkey Point Provincial Park, Ontario, Canada. J. Vector Ecol. 2007, 33, 64-69. [CrossRef]

93. Scott, J.D.; Durden, L.A. First isolation of Lyme disease spirochete, Borrelia burgdorferi, from ticks collected from songbirds in Ontario, Canada. N. Am. Bird Bander 2009, 34, 97-101.

94. Bouchard, C.; Beauchamp, G.; Nguon, S.; Trudel, L.; Milord, F.; Lindsay, L.R.; Bélanger, D.; Ogden, N.H. Associations between Ixodes scapularis ticks and small mammal hosts in a newly endemic zone in Southeastern Canada: Implications for Borrelia burgdorferi transmission. Ticks Tick-Borne Dis. 2011, 2, 183-190. [CrossRef]

95. Krakowetz, C.N.; Lindsay, L.R.; Chilton, N.B. Genetic diversity in Ixodes scapularis (Acari: Ixodidae) from six established populations in Canada. Tick Tick-Borne Dis. 2011, 2, 143-150. [CrossRef]

96. Ogden, N.H.; Koffi, J.K.; Pelcat, Y.; Lindsay, L.R. Environmental risk from Lyme disease in central and Eastern Canada: A summary of recent surveillance information. Can. Comm. Dis. Rep. 2014, 40, 74. [CrossRef] [PubMed] 
97. Simon, J.A.; Marrotte, R.R.; Desrosiers, N.; Fiset, J.; Gaitan, J.; Gonzalez, A.; Koffi, J.K.; Lapointe, F.-J.; Leighton, P.A.; Lindsay, L.R.; et al. Climate change and habitat fragmentation drive the occurrence of Borrelia burgdorferi, the agent of Lyme disease, at the northeastern limit of its distribution. Evol. Appl. 2014, 7, 750-764. [CrossRef] [PubMed]

98. Werden, L.; Barker, I.K.; Bowman, J.; Gonzales, E.K.; Leighton, P.A.; Lindsay, L.R.; Jardine, C.M. Geography, deer, and host biodiversity shape the pattern of Lyme disease emergence in the thousand islands archipelago of Ontario, Canada. PLoS ONE 2014, 9, e85640. [CrossRef] [PubMed]

99. Scott, J.D.; Anderson, J.F.; Durden, L.A.; Smith, M.L.; Manord, J.M.; Clark, K.L. Prevalence of the Lyme disease spirochete, Borrelia burgdorferi, in blacklegged ticks, Ixodes scapularis at Hamilton-Wentworth, Ontario. Int. J. Med. Sci. 2016, 13, 316-324. [CrossRef] [PubMed]

100. Keirans, J.E.; Litwak, T.R. Pictorial key to the adults of hard ticks, family Ixodidae (Ixodida: Ixodoidea), east of the Mississippi river. J. Med. Entomol. 1989, 26, 435-448. [CrossRef]

101. Wills, M.K.; Kirby, A.M.; Lloyd, V.K. Detecting the Lyme Disease Spirochete, Borrelia Burgdorferi, in Ticks Using Nested PCR. J. Vis. Exp. 2018, 132, e56471. [CrossRef]

102. Zinck, C.B.; Priest, J.M.; Shutler, D.; Boudreau, M.; Lloyd, V.K. Detection of Borrelia spp., Ehrlichia canis, Anaplasma phagocytophylum, and Dirofilaria immitis in Eastern Coyotes (Canis latrans) in Nova Scotia, Canada. J. Wildl. Dis. 2021, 57, 678-682. [CrossRef]

103. Nolte, O. Nucleic acid amplification based diagnostic of Lyme (neuro-) borreliosis-Lost in the jungle of methods, targets, and assays? Open Neurol. J. 2012, 6, 129-139. [CrossRef] [PubMed] 